\title{
RILIEVO MAGNETICO REGIONALE NELLE MARCHE PER LA ISTITUZIONE DI UN OSSERVATORIO MAGNETICO CENTRALE
}

\author{
M. Giorgi - E. Mrdi - C. Morelli
}

1. Premessa. - Uno dei compiti fondamentali dell'Istituto Nazionale di Geofisica è la realizzazione di una rete di osservatori magnetici per cui è necessaria innanzitutto la istituzione di un Osservatorio Magnetico di primo ordine nell'Italia Centrale. A questo fine da vari anni $\left(^{1}\right)$ l'I.N.G. ha compiuto campagne magnetiche sistematiche un po' dovunque, specialmente nel Lazio e nell'Umbria $\left({ }^{*}\right)$.

Per ovvie ragioni non è il caso di riportare nella presente nota i risultati in dettaglio avendo essi mostrato come queste zone si presentano in generale non del tutto favorevoli allo scopo.

Necessita infatti a questo fine una località pianeggiante, sufficientemente distante dai grossi centri abitati, dalle linee elettriche ad alta tensione, dalle ferrovie (specie se alimentate da corrente continua), e preferibilmente dal mare, in zona geologicamente non perturbata e esente da anomalie magnetiche locali (e possibilmente anche regionali).

$\mathrm{Ne}$ consegue subito che una località con queste caratteristiche è difficilmente reperibile nell'Italia Centrale dove in epoche geologiche anche recenti l'attività vulcanica è stata molto cospicua: per buona parte del versante Tirrenico, anche quando la geologia di superficie sembra essere favorevole, le misure magnetiche rivelano la presenza, a profondità generalmente esigue, di materiale vulcanico, presumibilmente depositato per trasporto eolico.

$\mathrm{Si}$ può dire inoltre che in tutto il restante territorio italiano, quando si escludano poche zone che sono d'altra parte da scartare per ragioni di disturbi artificiali già presenti o possibili a verificarsi in un immediato futuro (per industrializzazione od elettrificazione di linee ferroviarie od altre cause), non è facile trovare una località soddisfacente ad ogni necessario requisito per la fondazione di un Osservatorio magnetico di prim'ordine. L'Italia infatti è una terra geologicamente molto tormentata e varia dal punto di vista cronologico e litologico; il suo complesso aspetto geologico è fortemente complicato

(*) Fra le zone oggetto delle indagini compiute da E. Medr, C. Morelli ed altri sono: Fondi, Lenola, Genazzano, Magliana Sabina, Subiaco, Spoleto, Lago Trasimeno, ecc. 
dai vivacissimi fenomeni tettonici, verificatisi negli ultimi periodi geologici, che hanno determinato spesso una struttura morfologica molto movimentata.

In definitiva, le carte geologiche, i suggerimenti dei geologi, e l'esame della carta (scala 1:2.000.000) in cui è rappresentata la componente orizzontale della perturbazione del campo magnetico terrestre nelle stazioni (assolute) di primo ordine dell'Istituto Geografico Militare $\left({ }^{2}\right)$, hanno indicato come regione consigliabile quella delle Marche. Questa regione, infatti, tra la parte occidentale mesozoica dell'Appennino, piuttosto complessa, ed il monte Conero (m 572), che costituisce l'estremo lembo orientale del corrugamento secondario e terziario, presenta una vasta zona collinosa pliocenica di grande uniformità litologica e tettonica. Le formazioni plioceniche del Piacenziano (per la maggior parte) e dell'Astiano, con stratificazione quasi orizzontale o di poco inclinata, occupano quasi tutta la fascia litoranea fra Senigallia ed Ascoli Piceno fino ad una profondità di $20-30 \mathrm{~km}$ dalla costa. La forte erosione delle acque torrentizie e di precipitazione, molto intensa nell'era quaternaria, agendo su questi terreni pliocenici poco coerenti ha formato una monotona successione di più o meno dolci rilievi collinosi (al di sotto di circa $600 \mathrm{~m}$ ), sviluppatisi fra un corso d'acqua e l'altro, con andamento parallelo man mano degradanti verso il mare. Il Piacenziano, per la minor resistenza di questi terreni argillosi all'azione abrasiva, arriva solo fino ai $300 \mathrm{~m}$ sul livello del mare, mentre l'Astiano supera anche i $400 \mathrm{~m}$. Tra un rilievo e l'altro, nelle valli e sui fianchi delle colline specialmente lungo il corso medio e inferiore dei fumi, si estendono $i$ terreni quaternari costituiti da depositi fluviali. I terreni pleistocenici si sviluppano in piani con pendenza più o meno regolare verso il fondo delle valli, di preferenza a sinistra dei corsi d'acqua; più grande estensione hanno lungo l'asse vallivo le formazioni oloceniche (alluvioni fluviali, detriti di falda) di potenza da pochi metri fino a 40-50, specialmente verso il termine dei corsi d'acqua $\left({ }^{3}\right)$.

Da tutta la fascia pliocenica sopraddetta vanno scartati ai fini della ricerca una fascia di circa $7-10 \mathrm{~km}$ lungo la costa, anche per la ferrovia litoranea elettrificata, ed altre fascie all'incirca della stessa profondità a cavallo delle linee ferroviarie trasversali. Sicché in definitiva rimangono utilizzabili nella zona alcune " isole " omogenee, del diametro dell'ordine di una ventina di $\mathrm{km}$, nelle quali appunto si mostrava consigliable l'esplorazione magnetica.

Nel mese di giugno dello scorso anno fu eseguita una campagna 
di prima esplorazione magnetica specialmente in due zone, ritenute tra le più opportune, situate a nord e a sud della linea ferroviaria elettrificata Fabriano-Jesi-Falconara; precisamente le due zone sondate comprendono una fascia della regione geologicamente omogenea pliocenica sopraddetta, larga una diecina di chilometri con asse in direzione NNW-SSE che a nord va da Corinaldo, Belvedere Ostrense, fino a Iesi e a sud da Iesi, Torre di Iesi, Filottrano, fino a Montefano. I terreni maggiormente sondati con un infittimento delle misure furono ovviamente quelli più eccentrici rispetto alla linea ferroviaria elettrificata.

Le misure, eseguite con la bilancia verticale Schmidt n. 300075, avente una sensibilità di oltre $10 \gamma$ per divisione, confermarono la bontà della scelta e dettero modo di individuare le zone più adatte agli scopi della ricerca in due regioni aventi press'a poco come centro: la prima Belvedere Ostrense, la seconda Filottrano, rispettivamente a nord e a sud dell'Esino.

Fu deciso allora di eseguire una campagna di misure sistematiche e definitive in tutta la fascia litoranea, comprendente cioè non solo le regioni sopraddette ma anche le zone geologicamente omogenee e identiche alle precedenti a nord dal Tenna fino al Chienti e a sud del Tenna fino all'Aso.

Successivamente queste misure vennero estese verso nord per allargare ed infittire la rete di osservazioni nella zona di Corinaldo arrivando a comprendere il territorio a sud ed a nord lungo la valle del Cesano.

I risultati che qui sotto riportiamo si riferiscono appunto a questa campagna che si è svolta in due tempi: nel dicembre 1949 e per estendere la zona studiata sulla base di queste prime risultanze nel marzo 1950. Un'ulteriore breve estensione ad $W$ di Corinaldo venne eseguita nel maggio 1950.

Di preferenza le misure sono state eseguite sulle creste collinose evitando per lo più il fondo-valle dove il terreno di riporto, di potenza molto variabile da punto a punto, è generalmente impuro ed eterogeneo per la sua stessa natura.

I criteri eseguiti sono stati quelli già esposti in altro lavoro $\left({ }^{4}\right)$, limitando le misure alle variazioni della sola componente verticale $Z$ in quanto ciò era sufficiente per la ricerca in oggetto.

2. Strumenti. - Sono state adoperate tre bilance magnetiche di Schmidt, di cui una con testa di registrazione ha costituito l'Osser- 
vatorio per la variazione diurna e per l'indicazione di eventuali tempeste magnetiche, che ha funzionato con continuità dal dicembre 1949; alla fine di marzo 1950 venne inoltre messa in funzione in località diversa una seconda bilancia con testa di registrazione, secondo quanto si dirà al $\S 4$. Gli equipaggi magnetici erano tutti compensati per la temperatura, ad eccezione di quello della prima bilancia registratrice che era del vecchio tipo; ma ciò non ha avuto alcuna conseguenza essendo stato sistemato in un ambiente dove la temperatura controllata con un termografo è rimasta praticamente costante. Le massime escursioni diurne (differenze tra massima e minima) della temperatura che sono state registrate in detto ambiente non hanno mai oltrepassato $5{ }^{\circ} \mathrm{C}$; sensibilmente minori si sono poi mantenute nei giorni delle prime due campagne (v. § 3 ).

Prima di iniziare la campagna, con tutte le dovute cautele e norme raccomandate allo scopo $\left({ }^{5}\right)$, sono state eseguite sugli equipaggi magnetici le seguenti operazioni:

a) Aggiustamento per la latitudine. Richiedendosi, almeno a priori, una precisione notevole (v. $b$ ), si è preferito non operare con i magneti ausiliari, ma variare piuttosto l'aggiustamento in latitudine degli equipaggi. Nel nostro caso, essendo essi aggiustati per latitudini inferiori, ciò si è ottenuto spostando (di circa $1 / 8 \mathrm{di}$ giro) verso l'esterno il contrappeso situato verso il polo sud dell'equipaggio. Data l'esiguità di tale spostamento, esso non ha alterato la compensazione per la temperatura.

b) Aumento della sensibilità. Dato lo scopo per cui doveva servire il rilievo e dato che si doveva operare in una zona che a priori si poteva ritenere normale, bisognava mettersi in condizione di poter rivelare ogni minima anomalia. Invece la sensibilità di due degli equipaggi era piuttosto ridotta (circa $100 \% /$ divis.). Il loro contrappeso inferiore è stato quindi elevato al massimo, e si è ottenuta così una sensibilità dello stesso ordine di grandezza del terzo equipaggio (v. c).

c) Taratura. E stata eseguita con la bobina di Helmholtz per diversi valori dell'intensità di corrente; si sono ottenuti i seguenti

\begin{tabular}{|c|c|c|c|c|}
\hline & Fquipaggio & $\begin{array}{c}\text { dicembre } \\
1949\end{array}$ & $\begin{array}{c}\operatorname{maryo} \\
1950\end{array}$ & $\begin{array}{c}\text { maggio } \\
1950\end{array}$ \\
\hline n. 949 & (bilancia n. 300076) . . . . & $11,58+0,08$ & $10,25+0,06$ & $12,18 \div 0,08$ \\
\hline n. 646 & (bilancia n. 98570). . & $11,70+007$ & $12,89+0,08$ & $12,76+0.06$ \\
\hline n. 849 & (registratore n. 97851) . & $1,76 \div 0,01$ & $1,76+0.01$ & $2,21+0,02$ \\
\hline n. 1041 & (registratore n. 300075 ). & - & $5,91+0,07$ & $4,96 \div 0,02$ \\
\hline
\end{tabular}




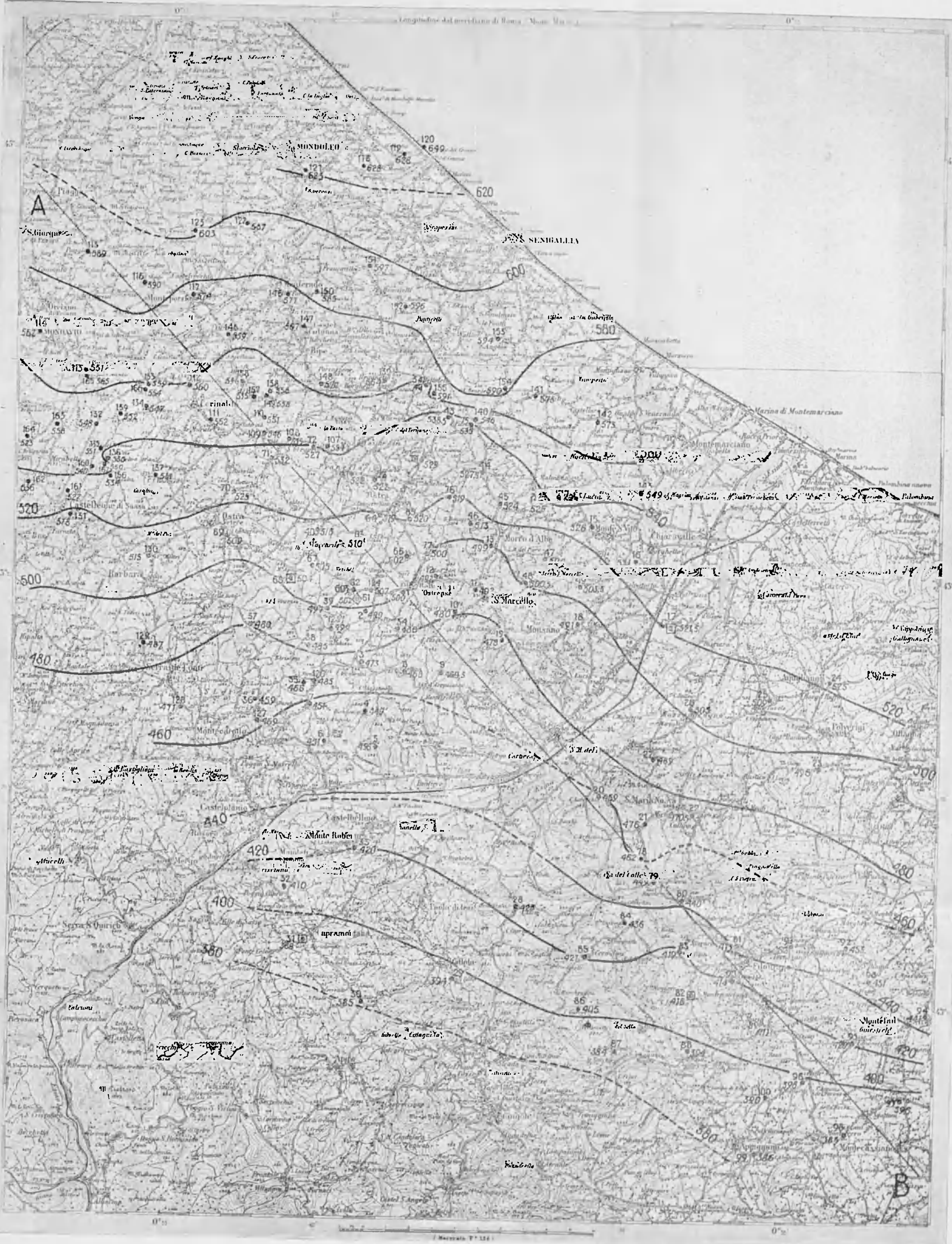

Fig. 1 - Distribuzione delle stazioni e valori convenzionali $($ n. $1=500 \gamma$ ) ridotti per la variazione diurna, nella zona di Jesi. 
valori per le costanti di scala $\varepsilon_{z}$ dei tre equipaggi $(\gamma /$ divis. per le bilancie a lettura diretta, $\gamma / \mathrm{mm}$ per le bilancie registratrici).

Con un magnete ausiliario, sono stati poi determinati $i$ segni per le bilancie registratrici, e controllati per le altre due bilancie per verificare se effettivamente ad un aumento del valore della componente verticale del campo $Z$ corrispondeva un aumento delle letture alla scala.

Dato che (soprattutto per ridurre al minimo gli errori di orientamento e di livellamento) nell'eseguire la campagna è stato poi seguito il metodo delle quattro letture (sinistra, destra, destra, sinistra), si è voluto determinare il grado di precisione con esso compatibile (per i valori sopraindicati delle costanti di scala). A questo scopo, sono state eseguite sulle due bilancie destinate ad operare in campagna, due serie di 10 determinazioni complete col metodo sopra indicato. Ne sono risultati $i$ seguenti errori medi:

$$
\begin{gathered}
\text { bilancia } \text { n. } 98570: \pm 1,57 \gamma \text {; } \\
\text { » } 300076: \pm 1,82 \gamma .
\end{gathered}
$$

Si conclude che, facendo astrazione da altre cause di errore (non comprese in quelle ora indicate), le bilancie adoperate possono garantire $i$ valori osservati entro $5 \gamma$. Le differenze fra di essi possono essere garantite quindi entro $+7 \gamma$. Risultato soddisfacente se si pensa che si tratta di bilancie ad equipaggi che hanno già un lungo stato di servizio, talvolta anche in condizioni tutt'altro che ideali.

Naturalmente qui si fa astrazione dagli errori di riduzione e principale fra questi la variazione diurna, del cui influsso parleremo al paragrafo 4.

3. Operazioni di campagna. - a) La prima campagna è stata eseguita dal 6 all'11 dicembre 1949. Onde consentire uno svolgimento rapido delle operazioni, sono state costituite due squadre indipendenti, ognuna delle quali ha operato secondo circuiti opportunamente scelti e studiati preliminarmente sulle carte topografiche. In ogni circuito, le stazioni venivano eseguite a distanze variabili da $l$ a $5 \mathrm{~km}$ l'una dall'altra, a seconda che si trattava di un raffittimento o di un primo rilievo a carattere regionale.

In ogni stazione le misure venivano eseguite in almeno due punti, distanti l'uno dall'altro da 10 a $50 \mathrm{~m}$, onde controllare la " normalità ) della stazione stessa. I valori riportati nelle tabelle che seguono sono pertanto la media di almeno 8 letture.

Sono state seguite sempre tutte le norme prescritte $\left({ }^{5}\right)$; in particolare l'orientamento è stato sempre curato entro il mezzo grado 
ed il livellamento entro mezza suddivisione delle livelle. Specialmente quest'ultima condizione si è ancora una volta dimostrata essenziale data la grande sensibilità; infatti per es., per uno scostamento della bolla di una sola suddivisione nel livellamento si sono riscontrate differenze nelle letture di ben $8 \div 10 \gamma$.

Durante il rilevamento eseguito in questo primo periodo il cielo quasi sempre coperto ha facilitato molto la pratica realizzazione di questa condizione. Ma anche negli altri momenti lo strumento è stato sempre protetto dai raggi diretti del Sole, perché si è nuovamente constatato che l'irraggiamento rende quasi impossibile il mantenimento di una livellazione corretta, srettificando quasi subito le livelle. Per questo motivo, e per il fatto che l'escursione diurna esterna della temperatura è stata quasi sempre limitata a $7 \div 8{ }^{\circ} \mathrm{C}$ al massimo, la stagione in cui si è operato può essere considerata tutt'altro che inadatta per tali campagne.

Complessivamente sono state eseguite 106 stazioni (non comprendendo in questo numero le indispensabili ripetizioni e numerose stazioni eseguite in fondo valle $o$ in relativa prossimità di centri abi: tati, o di linee di conduzione elettriche o di linee ferroviarie elettriche onde stabilire una approssimativa valutazione delle relative perturbazioni). La loro successione è indicata nelle figure 1 e 2 , dalle quali risulta pure chiaramente l'ordine dei circuiti. Tutti i dati che ad esse si riferiscono, sia per le osservazioni che per le riduzioni, sono riportati nelle tabelle I e II allegate.

Punto di partenza è stata la stazione n. 1 , a SW di Belvedere Ostrense (v. fig. 1), alla quale è stato attribuito convenzionalmente il valore di 500 per le letture. Le letture medie $L_{m}$ riportate nelle tabelle I e II sono appunto tutte riferite a questo valore convenzionale.

Nelle tabelle I e II i riferimenti sono effettuati rispetto alla carta 1:100.000 dell'Istituto Geografico Militare, da cui sono ricavate pure le figg. 1 e 2.

b) La seconda campagna è stata eseguita nei giorni 27-29 marzo 1950 , con le stesse norme della precedente. Sono state eseguite altre 52 stazioni, oltre alle varie ripetizioni indicate nella tabella III. Altre numerose stazioni sono state escluse perchè eseguite in giorni troppo perturbati, sicché non sarebbe stato possibile applicare una esatta correzione diurna, secondo quanto si dirà al $\S 4$. La loro distribuzione risulta dalla fig. 1 .

Inoltre nelle zone che più parevano indicate per la scelta del posto dell'Osservatorio (stazioni n. 107, 124, 125, 136, 156, 157, 167) 


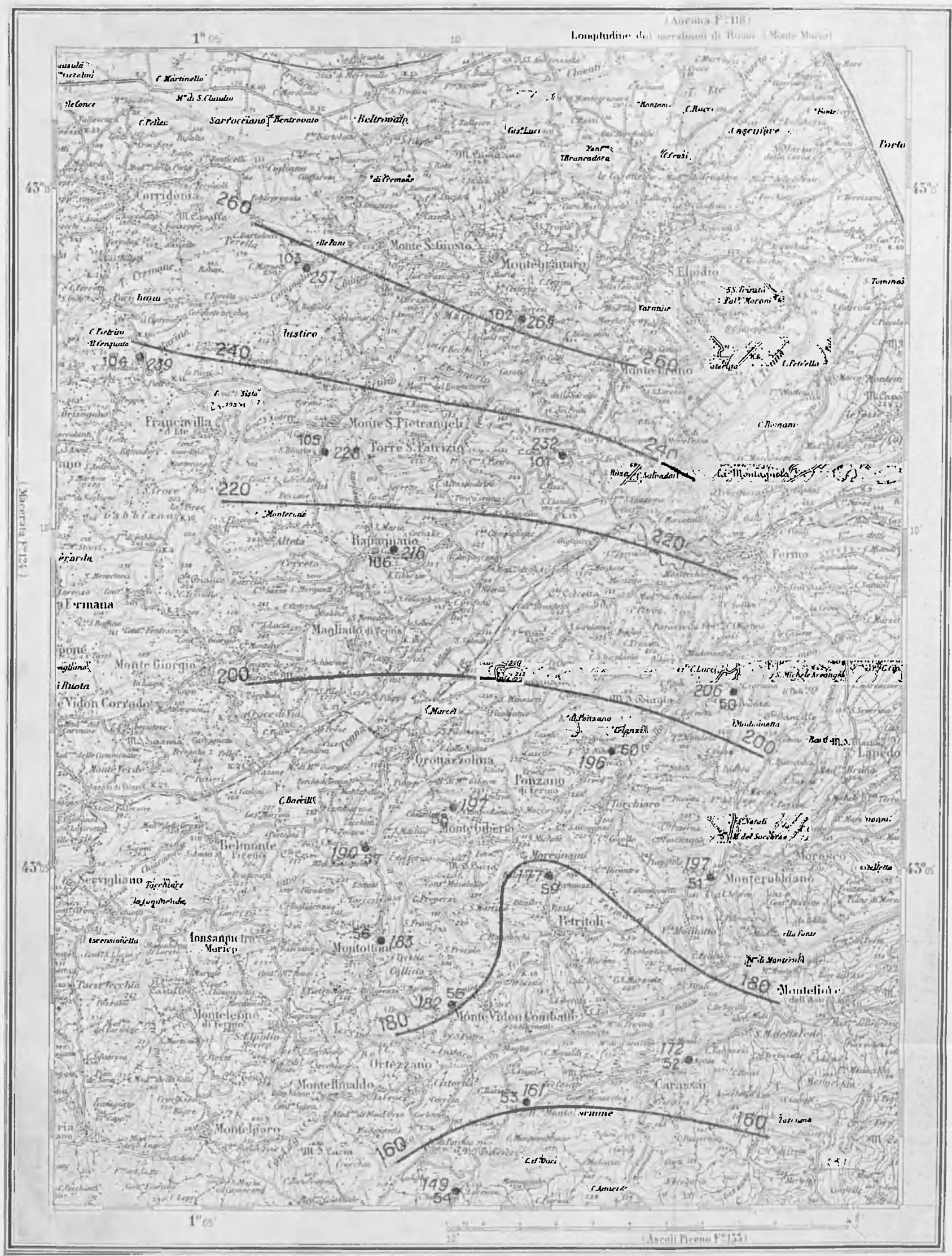

Fig. 2 - Distribuzione delle stazioni e valori convenzionali (n. $1=500 \gamma$ ) ridotti per la variazione diurna, nella zona di Fermo 
TABELLA I? : STAZIONI MAGNETICHE NEL TERRITORIO DI JESI -(f.117), ESEGUITE NEL DICEM.1949-

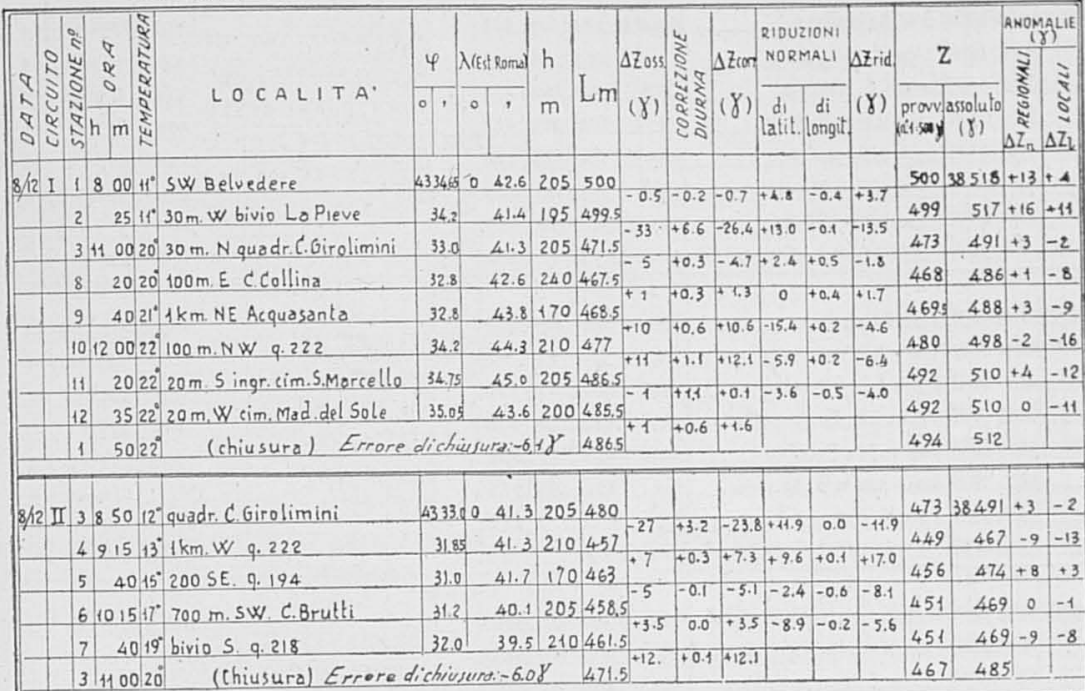

8/2 III $11135519^{\circ} 20$ m. S ingr cim. S. Marcello 4334.30 $045.0205483 .5+12 \quad-4.8+7.2-11.3+0.2-4.249238510+4-12$ $1.141020^{\circ}+\mathrm{km}$. SW Morro

12520 bivio SE. S. Amico $4020^{\circ} 1 \mathrm{~km}$. NW. Monte S.Vito $115.0519500 \mathrm{~m}$. Nw. C. Marinello

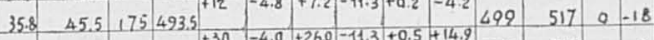
36.8 $47.05165515 .5 \quad 525 \quad 543+15-7$ $36.2 \quad 48.6 \quad 150518 \underbrace{+3}_{+6}-2.0+1.0+6.0+0.5+7.4526 \quad 544+23-3$ $35.4550 .255(524)^{8}-6{ }^{+6}-1.7+4.3+8.4+0.6+13.3531 \quad 549+37+6$ $4018^{\circ} 1$. G.M n? 760 : Villa Piano Ameno 1160017200 m.S.Cim.S.Maria 2017700 m.S. 9.180

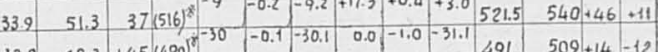

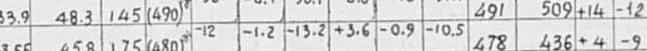

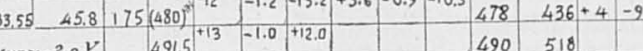
$113517^{\circ}$ (chiusura) Errore dichiusurd:-2.0\% $491.5^{\circ}$

2/12 IV $117.4012^{\circ} 20 \mathrm{~m}$. S. ingr. Cim. S. Marcello 4334730 $4502054965-32-1.5-33.5+53.6+1.4+16.549238510+4-12$ $20.8 .504^{\circ} 3 \mathrm{~km}$. SE, Jesi

$2112.4518^{\circ}$ bivio $2 \mathrm{~km}$. SW. 5 Maria $\mathrm{N}$. $2814.0017^{\circ}$ bivio $1 \mathrm{~km}$. W. C. Costa $29.949 .1100(470)^{4}+10.5+7.2+17.7+6.5+0.5+24.7459 \quad 477+26-1$ 29.3 $50.55300(479)^{5} \quad-1.0-51.0+22.1-1.4-30.3 \quad 476 \quad 4.94+50+18$ $\begin{array}{lllll}27.3 & 46.3 & 290 & 436\end{array}$ $293015^{\circ} 200$ m. S. cim staffolo $\begin{array}{lllll}25.6 & 44.3 & 415 & 416\end{array}$ $3015.0514^{\circ} 100 \mathrm{~m}$.W. 9.562 3) $4515^{\circ}$ I.G.M. . . $7621 \mathrm{~km}$. S.W. $3216.1015^{\circ} 2 \mathrm{~km}$. 5.w. Moiolati $25.1 \quad 41.5 \quad 530410$ $26.5 \quad 39.5 \quad 420413$ $27.7 \quad 38.95380(433)^{8}$ $332515^{\circ} 500$ m.S.E. M ${ }^{\text {IR Roberto }} 28.65$ 41.15 300442 $11171015^{\circ}$ (chiusura) Erroredichiusurg: $-3.3 \gamma$ \$ 5015 \begin{tabular}{llllllll}
-50 & -1.0 & $-51.0+22.1$ & -1.4 & -30.3 & 425 & $443+20+1$ \\
\hline 23 & -8.8 & $-31.8+18.5$ & -0.7 & -14.0 &
\end{tabular} \begin{tabular}{l|l|l|l|l|l|l}
-6 & -2.9 & $-8.9+5.4+1.0+4.5$ & 394 & $412+7$ & -6 \\
\hline
\end{tabular} $\begin{aligned} & 3.5-0.3+3.2-15.4-0.7-12.9 \\ & 388\end{aligned} \quad 403+2-2$ \begin{tabular}{l|l|l|l|l|l|l|l}
+23 & $-0.5+22.5$ & -13.0 & $-0.2+93$ & 388 & $406+11-10$
\end{tabular}

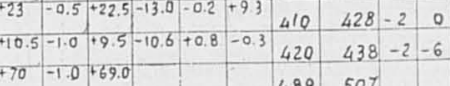
489507

$9 / 2$ Y $219204^{\circ}$ bivio $2 \mathrm{~km}$.SW.S.MariaN. $43293050.55300(474)^{*}-6+0.1-5.9-3.0+0.8-2.147638494+50+18$ $22 \quad 45.14^{\circ} 1 \mathrm{~km}$ SW C?"Monti. $29.6 \quad 53.0 \quad 215 \quad 469+26-0.2+25.8-10.6+1.1+10.3 \quad 470.5 \quad 489+43+4$

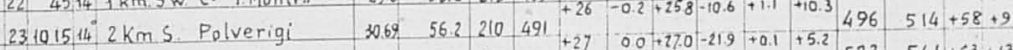

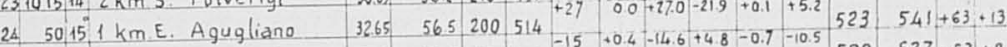

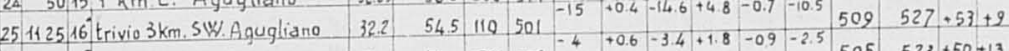

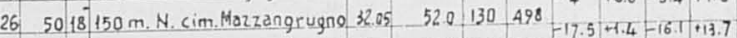
$27122018^{\circ} 2 \mathrm{~km}$. NW. S.Maria N. $\quad 30.8 \quad 50.75$. $180 \quad 483$ $4518^{\circ}$ (thiusural Errore dichiesure $+5.1 \gamma \quad(675)^{*}$ $\begin{array}{lll}-9+1.3-7.7 & 481.5500\end{array}$ 


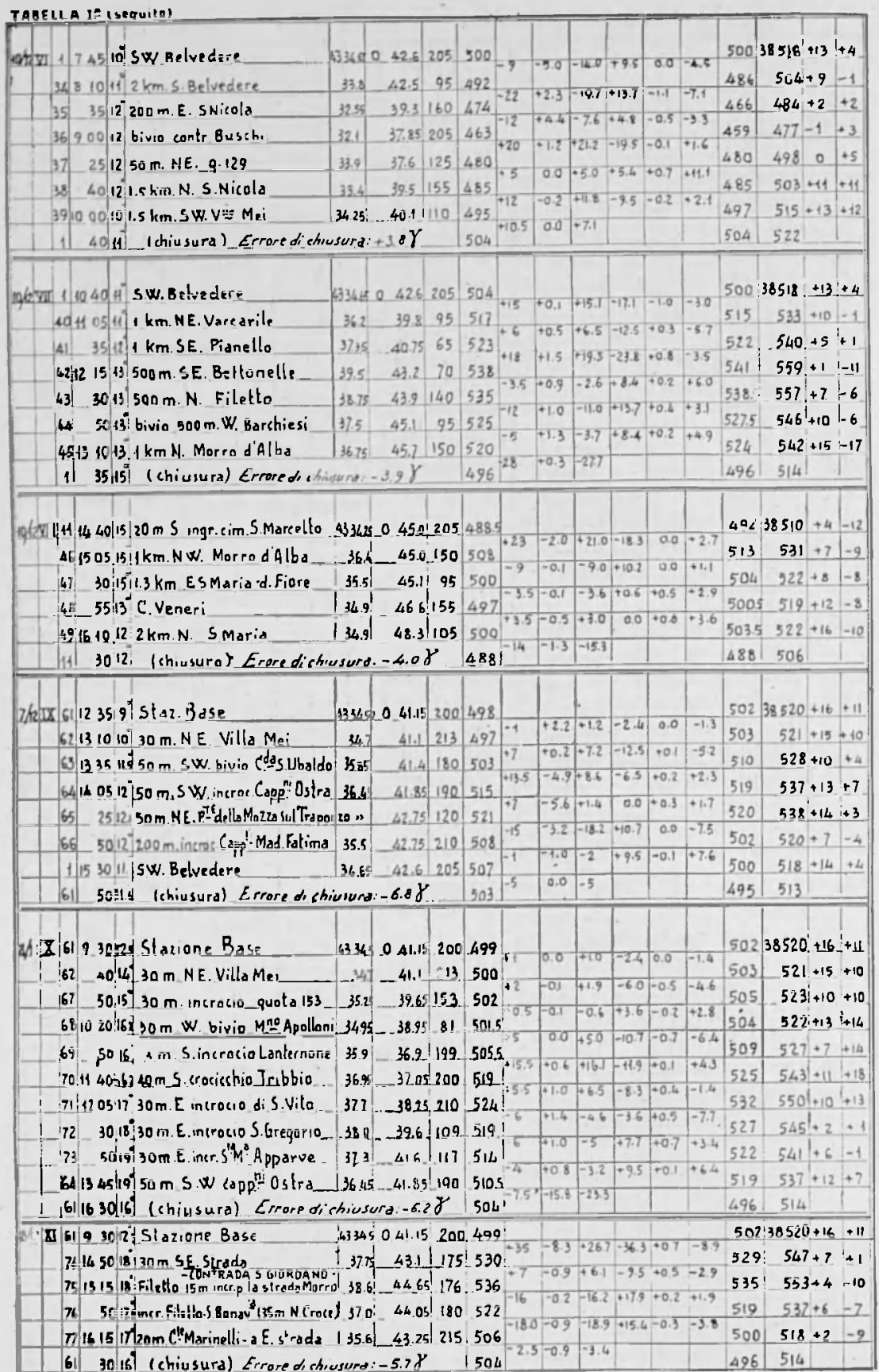


TABELLA I: (seguito)

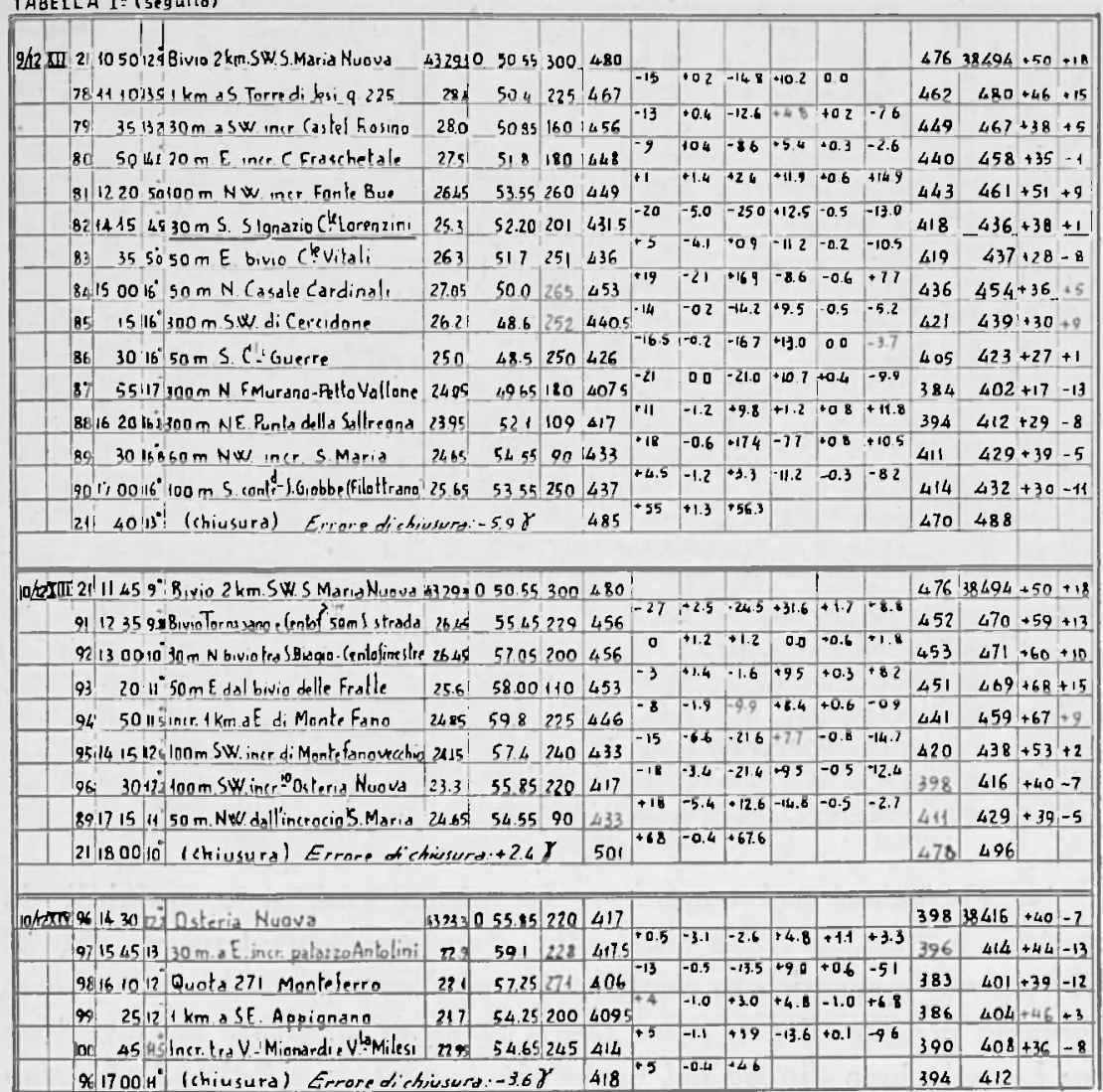

sono stati eseguiti dei raffittimenti di dettaglio, secondo gli schemi topografici di cui alla fig. 3 .

c) Il 7 maggio 1950 , in occasione di una revisione alle stazioni registratrici, sono state eseguite altre 12 stazioni, con l'estensione di un raffittimento di dettaglio alla Croce del Termine (stazione n. 167). Essendo risultata questa giornata alquanto perturbata è stato riportato un solo circuito di 12 stazioni per le quali è stato possibile applicare accuratamente la correzione per la variazione diurna. Esse sono riportate nella tabella III e indicate nella fig. 1 .

Complessivamente quindi sono qui riportati i valori di 170 stazioni, mentre in tutto se ne sono eseguite oltre 400 , senza computare le ripetizioni e $\mathrm{i}$ raffittimenti. 
TABELLA IA . STAZIONI MAGNETICHE NEL TERRITORIO DI FERMO $\left(f_{\text {.125 }}\right.$, ESEGUITE NEL DICEM. 1949

\begin{tabular}{|c|c|c|c|c|c|c|c|c|c|c|c|c|c|c|c|c|c|}
\hline \multirow{3}{*}{$\begin{array}{l}7 \\
2 \\
0\end{array}$} & \multirow{3}{*}{ 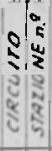 } & \multirow{3}{*}{ 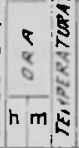 } & \multirow{3}{*}{ LOCALITA' } & \multirow{3}{*}{$\frac{\varphi}{0.1}$} & \multirow{2}{*}{$\lambda(t, 1) \mathrm{ln})$} & \multirow{3}{*}{$\begin{array}{l}h \\
m\end{array}$} & \multirow{3}{*}{ Lm } & \multirow{3}{*}{$\begin{array}{c}\Delta Z_{\text {oss }} \\
(Y)\end{array}$} & \multirow{3}{*}{ 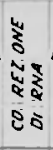 } & \multirow{3}{*}{$\begin{array}{l}\Delta Z_{(0 r} \\
\left(X_{1}\right)\end{array}$} & \multirow{2}{*}{\multicolumn{2}{|c|}{$\begin{array}{l}\text { RIDUZIONI } \\
\text { NORMALI }\end{array}$}} & \multirow{3}{*}{$\begin{array}{l}\text { azrid } \\
\text { (J) }\end{array}$} & \multirow{2}{*}{\multicolumn{2}{|c|}{ Z }} & \multicolumn{2}{|c|}{$\begin{array}{c}\text { ANDN } \\
18)^{2}\end{array}$} \\
\hline & & & & & & & & & & & & & & & & & \\
\hline & & & & & 0 & & & & & & totut & longlil & & $\pi s=5 d y$ & $\begin{array}{l}\text { ssogiulo } \\
(Y)\end{array}$ & o & $\begin{array}{r}0 \\
0 \\
\Delta z \\
\Delta z\end{array}$ \\
\hline \multirow[t]{12}{*}{$H / 2 x$} & W 50 & 113012 & $150 \mathrm{~m}$ S.w. C Fortuna & [3 07. & 115.65 & 100 & 244.5 & & & & & & & 206 & 38228 & +27 & $\cdot$ \\
\hline & .51 & 120016 & $1 \mathrm{~km}$. Sw Monterubbiano & as: & 152 & 310 & 237 & & & 83 & +178 & 22 & $\cdot 21.3$ & 197 & 10,5 & .18 & - \\
\hline & 52 & $-30.16^{\circ}$ & SAoala & 02 & 148 & 290 & $21 \mathrm{~A}$ & -27 & +1.8 & -252 & +31.0 & 1.01 & +5.7 & 172 & 190 & .54 & - \\
\hline & 53 & $45.16^{\circ}$ & $100 \mathrm{~m} \mathrm{~N}$. RuM Vormine & ots & 11.5 & 320 & 204 & 12 & .08 & -11 & 160 & -1.1 & 63 & 161 & 179 & +48 & - \\
\hline & 20 & 131016 & $500 \mathrm{~m}$ SW. Grose SLerenzo & 001 & 101 & 320 & 192 & & & & $\| \mathbf{L T}$ & .05 & -1.3 & 149 & 167 & .50 & $\cdot$ \\
\hline & 59 & 3516 & $50 \mathrm{~m}$ N. verehio cim Collina & 034 & 100 & 305 & 220 & & & 9331 & -110 & 00 & +21 & 182 & 200 & .52 & $\cdot$ \\
\hline & 36 & 6140016 & $1 \mathrm{~km}$. N. sim Montollor & os & 083 & 255 & 223 & & & & 101 & & & 183 & $2 Q 1$ & \pm 1 & $\cdot$ \\
\hline & 51 & 1516 & Serp $3 \mathrm{~km} N$. Montottone & os: & 083 & 130 & 234 & & & & & & & 190 & 208 & +34 & - \\
\hline & 52 & $3516^{\circ}$ & i $\mathrm{km} \mathrm{Nw.M-Giberlo}$ & os: & 100 & 160 & 2435 & & & & & & & 197 & 215 & +36 & - \\
\hline & 59 & 5517 & $1 \mathrm{~km}$ sw Moregnano & ard & 11.9 & 300 & 228 & & 07 & & & & -317 & 177 & 195 & +26 & $\cdot$ \\
\hline & 60 & $1520,16^{\circ}$ & $1.5 \mathrm{~km}$. N. Torchioro & $\boldsymbol{\omega} . \mathbf{t}$ & 13.2 & 175 & 244 & & & & -148 & & 1.0 & 196 & -214 & +26 & - \\
\hline & 50 & $40: 15$ & (chissura) Errorea & a thiv & usu of $-1,21$ & & 252 & & -01 & +8.9 & & & & 204,5 & 538223 & & $\cdot$ \\
\hline 14/25 & & & $\begin{array}{l}50 \mathrm{~m} \text { W Bivio Torres Patrizio. } \\
\text {-MONTE UMANO- }\end{array}$ & Is $14 !$ & 112.2 & 152 & 316 & & & & & & & 232 & 38350 & +14 &. \\
\hline & ant & $2: 13 \times 9.12$ & Eivio Madonno della Croce & & 2. 1135 & 148 & 343 & & & & $=0$ & -03 & +96 & 265 & 283 & +23 & $\cdot$ \\
\hline & - 1 & $3,1300,135$ & Bivio di Campiglia & 134 & -215 & 230 & 334 & & +1.5 & & -65 & -1.4 & -164 & 257 & 275 & +7 & - \\
\hline & 108 & 2516 & Bivio di Hacina & 121 & 3.7 & 203 & 318 & & +1. & 7.6 & +4.8 & -12 & -4.5 & 359 & 2.57 & +2 & $\cdot$ \\
\hline & 108 & $50 \sin ^{\circ}$ & $1 \mathrm{~km} .5 \mathrm{~d}$ MTIS.Pelranaeli & 11.21 & 2.65 & $2 \leq 0$ & 310 & & & -10.7 & +14.3 & 11.3 & +4.9 & 228 & 246 & +7 & - \\
\hline & a & 14 sohis: & Insro:10 $800 \mathrm{~m}$. E di Rapaqnano & 96 & 88 & 300 & 300 s. & & $\begin{array}{c}-10.1 \\
0.1\end{array}$ & -12.1 & -173 & 0.5 & .5 .7 & 216 & 234 & +13 & - \\
\hline & ol & 15 ogik & (chiusurs) - Errosed $d$ & 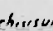 & ure $-2 x y$ & & 320 & +13 & -2.7 & +10.3 & & & & 326 & $24 \Delta$ & & - \\
\hline
\end{tabular}

4. Variazioni nel tempo. - In misure cosi poco variabili da stazione a stazione come quelle che dovevamo attenderci durante il rilevamento magnetico in questione in una zona presunta " normale", abbiamo pensato di non poter fare a meno di applicare la correzione per la variazione diurna nel modo più accurato possibile ed abbiamo provveduto quindi all'impianto di una registrazione continua delle variazioni della componente $Z$ del campo.

La prima bilancia registratrice è stata sistemata il 6 dicembre 1949 in una vecchia fattoria ora disabitata, circa $500 \mathrm{~m}$ a sud di Villa Mei; in prossimità della fattoria è stata fissata la stazione base n. 61 (v. fig. 1). La località è del tutto isolata, in aperta campagna e solo una volta al giorno il custode vi si reca per il cambio della carta fotografica.

Il registratore tipo (I.N.G. » costruito nelle officine dell'Istituto Nazionale di Geofisica, ha funzionato per i primi due giorni con velocità di $10 \mathrm{~mm} /$ ora; successivamente questa è stata aumentata a 16,5 min/ora poi ulteriormente e definitivamente a $30 \mathrm{~mm} /$ ora. Ciò soprattutto per mettere in evidenza le variazioni a breve periodo di cui ora parleremo.

La variazione diurna si è rivelata anche qui incostante. 


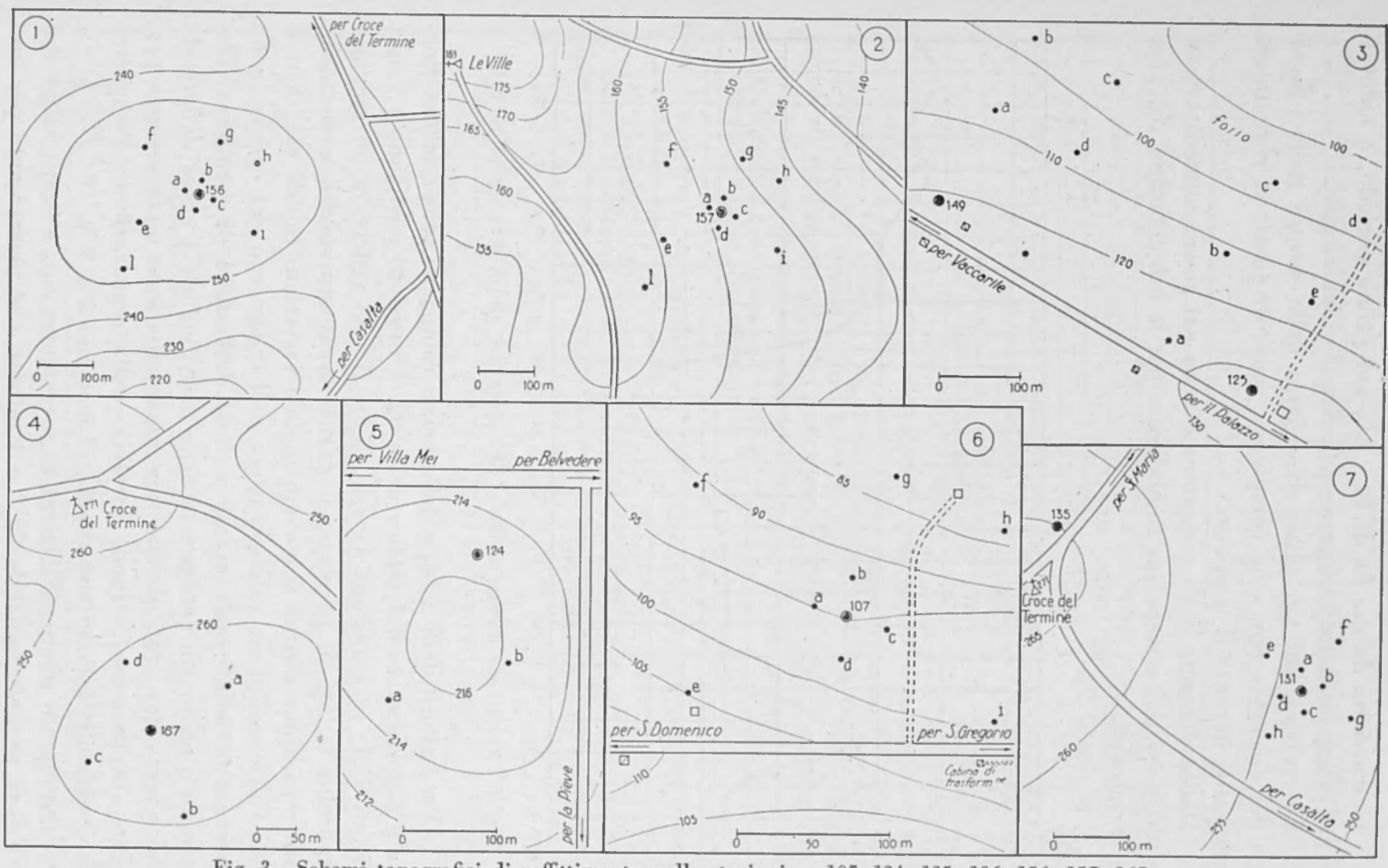

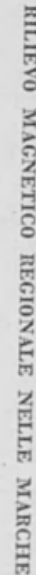

Fig. 3 - Schemi topografici di raffittimento nelle stazioni n. 107, 124, 125, 136, 156, 157, 167. 
L'escursione diurna fu di $20 \gamma$ ca., nel primo periodo, e notevolmente maggiore nelle campagne del marzo e del maggio.

Le registrazioni nei primi due giorni delle misure nella campagna del dicembre non sono complete a causa di guasti al registratore verificatisi durante il trasporto.

Dall'andamento della variazione diurna nei giorni immediatamente successivi si è estrapolato l'andamento per le ore mancanti nei giorni incompleti.

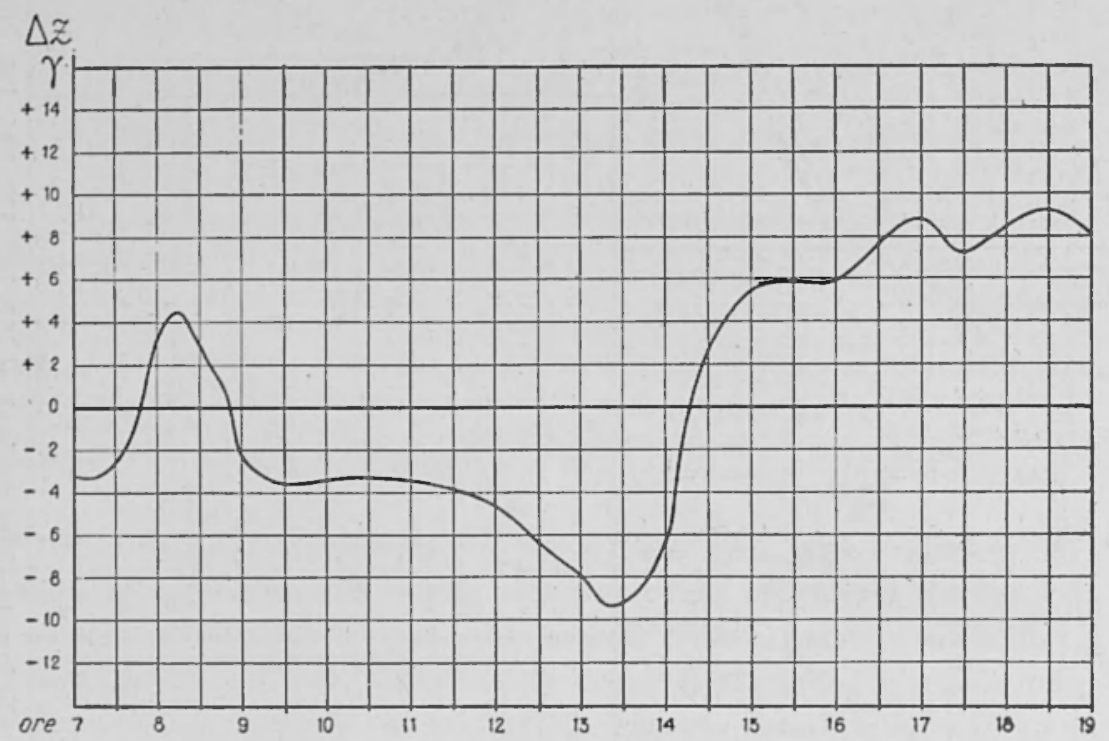

Fig. 4 - Variazione diurna media a Belvedere nei giorni 6-11 dicembre 1949

Per i giomi delle misure della prima campagna la variazione diurna è rappresentata dal grafico della fig. 4 ottenuto mediando i valori diretti delle registrazioni. Queste, come si può vedere in un esempio riportato in fig. 5, presentano infatti un aspetto molto complicato. Alla variazione diurna si sovrappongono variazioni rapide che denunciano una complessa microstruttura dell'andamento del campo. Sono facilmente visibili onde aventi periodo dell'ordine di grandezza d'un minuto o meno (in genere non bene individuabile a causa della scala dei tempi), che costituiscono una micropulsazione quasi sempre presente; rappresenta il fondo instabile della registrazione; l'ampiezza di questa oscillazione non supera al massimo 2 o $3 \gamma$.

Su queste microoscillazioni si sovrappongono, a tratti, altri impulsi, di periodo variabile da 2 a 3 minuti, che permangono per lore- 
TABELLA III : STAZIONI MAGNETICHE NEL TERRITORIO DI JESI -(\$.125) ESEGUITE NEI MESI DI MAR゚ MAGTIO IGSO

\begin{tabular}{|c|c|c|c|c|c|c|c|c|c|c|c|c|c|c|c|c|}
\hline \multirow{2}{*}{ 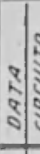 } & \multirow{2}{*}{ 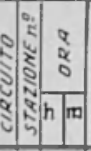 } & \multirow{2}{*}{ LOCALITA' } & \multirow{2}{*}{$\frac{4}{\circ}$} & Alistromes & \multirow{2}{*}{$\begin{array}{l}\mathrm{h} \\
\mathrm{m}\end{array}$} & \multirow{2}{*}{$\operatorname{Lm}$} & \multirow{2}{*}{ (0) } & \multirow{2}{*}{ 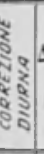 } & \multirow{2}{*}{$\begin{array}{l}\Delta z \text { zart } \\
(\gamma)\end{array}$} & \multicolumn{2}{|c|}{$\begin{array}{l}\text { RIDUZIONI } \\
\text { NORMALI }\end{array}$} & \multirow{2}{*}{$\begin{array}{l}\text { szrid } \\
(\gamma)\end{array}$} & \multicolumn{2}{|c|}{ Z } & \multicolumn{2}{|c|}{$\begin{array}{l}\text { MMOMALIE } \\
\text { (Y) }\end{array}$} \\
\hline & & & & - & & & & & & di & {$\left[\begin{array}{c}\text { dii } \\
\text { bngit }\end{array}\right.$} & & $\begin{array}{l}\text { prove: } \\
\text { nitsopp }\end{array}$ & if: & $\begin{array}{c}5 \\
\vdots \\
\vdots \\
\vdots \\
z_{n}\end{array}$ & 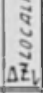 \\
\hline & 561753 & i) Staziene base & 4345 & 0415 & 200 & 525 & & & & & & & 502 & 38520 & -16 & +11 \\
\hline & 62,340 & i) villa Mei & $y_{-7}$ & 41.1 & 213 & 529 & & -6.3 & -1.3 & -2.4 & 0.0 & -3.7 & 501 & 519 & & +10 \\
\hline & 1079251 & S suegorio & 38.0 & 40.1 & 25. & 563 & & & & & & & 554 & 572 & & +27 \\
\hline & log. 40 & $4 !$ & & & 95 & $567^{\circ}$ & & +0.6 & +52. & & & & 560 & 578 & & \\
\hline & 107,55 & 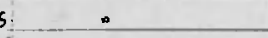 & & & 92 & $56 \mathrm{kS}$ & & 40.5 & -2.5 & & & & 557 & 575 & & \\
\hline & 107jo 00,1 & 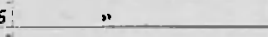 & & & 95 & 562 & & +1.8 & -1.2 & & & & 556 & 574 & & \\
\hline & (ior] 05 & 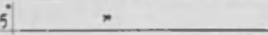 & & & 98 & 564 & & +4.6 & +2.1 & & & & 558 & 576 & & \\
\hline & 107.15 & $5^{\circ}$ & & & 105 & 565 & & +1.4 & +2.6 & & & & 561 & 579 & & \\
\hline & 1007,25 & $\Rightarrow$ & & & 21 & 564 & & 8 & $\cdot 0.8$ & & & & 561 & 579 & & \\
\hline & 107. 40 & $n$ & & & 83 & 566 & +3 & -0.4 & +2.6 & & & & $56 \mathrm{~A}$ & 582 & & \\
\hline & 10. 35 : & 5 & & & 84 & 567 & +1 & -5.7 & -4.7 & & & & 559 & $5 n$ & & \\
\hline & 3711105 & ? & & & 100 & 564 & & -3.1 & -7 & & & & 552 & 570 & & \\
\hline & 107. 15 & .. & & & 106 & 567 & +4 & -0.4 & +3.6 & & & & 556 & 574 & & \\
\hline & 10840 & 1. Trivia NW. SGregorio & 382 & 388 & 165 & 564 & -4 & 6 & -1.4 & -2.4 & -0.8 & -4.6 & 555 & 573 & +27 & +29 \\
\hline & $109 \quad 5017$ & 7i $500 \mathrm{~m}$. E. S.Domenico & 383 & 38.0 & 90 & 558 & & $=0.5$ & $|-2.5|$ & -1.2 & -0.3 & -10 & 546 & 564 & $4+17$ & +21 \\
\hline & $110: 12001$ & 1) quado sul Nevola & 38 तो & 380 & 50 & 561 & & $+1.4 \mid$ & +5.4 & $\mid-4.2$ & 0.0 & +1.2 & 551 & 969 & $+18+$ & +22 \\
\hline & $\operatorname{lin} \quad 15$ & 6́ bivio S.E. Corinaldo & 386 & 36.3 & 120 & 562 & & 0.0 & +1 & $-1 / 2$ & $-0.6 \mid$ & -0.8 & 552 & 570 & $1+17$ & -28 \\
\hline & 11225 & 6 bivin S. Coppuecini & 39d & 35.6 & 225 & 565 & 4 & -1.1 & -2.9 & -8.8 & $-0.2]$ & -6.1 & 555 & 573 & $3+11$ & +2 \\
\hline & Glit3 $\left.38\right|_{1:}$ & 3 (chiusura) Errarest & Shiers & tar.d: -50 & ay & 532 & & -37.6 & -80.6 & & & & & & & \\
\hline & 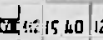 & 5. Capp & 43389 & $0 \_35.6$ & 225 & 576.5 & & & & & & & 560 & & & \\
\hline & 413 lb 000 & $4 . \mathrm{km}$. Nw. S.Michele & 39.25 & -32.2 & 30 & $i 576$ & -1 & -2.3 & 0.3 & -36 & -1.2 & -45 & 561 & 579. & +12 & +39 \\
\hline & 14210.1 & 4 Cimitero Mondavio & $406 !$ & -30.6 & 250. & 579 & & -28 & 51.2 & -9.5 & -0.5 & +2.5 & 562 & $580^{\circ}$ & $0+3$ & +31 \\
\hline & $115 \quad 3013$ & Trivio Poggio & 485 & 32.1 & 200 & 607 & & -8.9 & $-22.1+$ & -20.9 & 105 & +25.2 & 589 & 607. & $9+10$ & +33 \\
\hline & 116.4013 & ic C. il & $418:$ & -34.0 & 250 & 610 & & 8.3 & +0.7 & +77 & {$[+0.7$} & +9.1 & $590^{\circ}$ & 608 & $8+19$ & .37 \\
\hline & $11 \%-501$ & E. Monteporzi & 41.5. & -35.6 & $95:$ & 599 & & 1.2 & $-15.2]^{4}$ & 43.5 & {$[+0.6$} & -11.2 & 574 & 592 & $2+8$ & +20 \\
\hline & $1181710, k$ & $1 \mathrm{~km}$.SE. C. Tinti & $\angle 46$ & .411 & 20 & (636) & & +51 & +5.1 & $\mid-3137$ & H.9 & +213 & 628 & 646 & $5+29$ & .25 \\
\hline & iig $\quad 20 ! 14$ & i $200 \mathrm{~m}$.E C. Volpini & 44.9 & $42.3 i$ & 18 & $(636)^{(2)}$. & & +0.9 & +0.9 & $|-3.6|$ & +0.4 & -2.3 & 628 & 646 & $6+27$ & +19 \\
\hline & $120 \quad 3011$ & 4'Fore del Cesana & 45.1 & 43.1 & $|-1|$ & $(654)^{24}$ & & -2.3 & +20.7 & $-2.4 \mid$ & +0.3 & +186 & 649 & 667 & $1+45$ & +35 \\
\hline & i2!! 45 & $41 \mathrm{~km}$ SF Mondolfo & 465 & 39.2 & 70 & $(636)^{(1)}$ & -29 & -32 & -262 & +65 & -1.3 & -21.0 & 623 & 641 & 1.24 & +26 \\
\hline & $122.1800,14$ & Piag & 43.2 & 373 & 90 & 608 & 35 & -0.2 & -35.8 & +14.2 & $-07 i$ & $i^{-22.3}$ & 587 & 605 & $5+2$ & +10 \\
\hline & $123 \quad 15 \quad 14$ & $41.5 \mathrm{~km}$ E. M resceo & 430 & 35.61 & 150 & 621 & & -07 & 46.3 & 1.2 .4 & -0.6 & +18.1 & 603 & 621 & +20 & +32 \\
\hline & $112 \int^{4} \quad 40$ & 4) (shiusura) Errosed d & chiese & urd: $-3,9$ & $9 \gamma$ & 5815 & $1^{-51}$ & +40 & -47 & & & & 556 & 574 & $|+13|$ & \\
\hline & & & & & & & & & & & & & & & & \\
\hline & 74 61124510 & ione base & 43345 & 041.15 & 200 & 528 & & & & & & & 502 & 38520 & $1+16$ & +11 \\
\hline & $24815 \mathrm{n}$ & Campo Cinqolani & $34 ?$ & 41.6 & 215 & 536 & & -4.9 & +5.1 & -2.4 & +0.2 & +29 & 507 & 525 & 210 & +13 \\
\hline & 2511 & * & & & 215 & 43 & & -5.1 & +39 & & & & 511 & 529 & & \\
\hline & 3012 & " & & & 215 & 38 & & -18 & -78 & & & & 503 & 521 & & \\
\hline & 4012 & $30 \mathrm{~m}$ W. bivio Lo Pieve & 34.2 & 41.4 & 195 & 536 & & & -48 & +53 & -0.1 & .04 & 498 & 516 & +15 & +11 \\
\hline & 345516 & i $2 \mathrm{~km} . \mathrm{S}$. Belvedere & 33.8 & $\angle 2.5$ & 95 & 526 & & +37 & -9.3 & .4 .2 & +0.4 & -5.7 & 489 & 507 & +11 & -1 \\
\hline & 391015 & $=30 \mathrm{~m} \mathrm{~N}$ qudriv. C.Girolimini & 30 & 41.32 & 205 & 519 & & +3.2 & -5.8 & +89 & -0.4 & -27 & 483 & 501 & +14 & -2 \\
\hline & 125. 20,15 & ; $\mathrm{km} . N N W$. il Palazzo_ & 33.8 & -40.31 & 135 & 525 & & +2.6 & +10.6 & $-\infty$. & -0.3 & +1.4 & 494 & 512 & +15 & +13 \\
\hline & 125. 30,15 & $\rightarrow$ & & & & 523 & & +1.8 & $\begin{array}{ccc}-1 & 5 \\
-7 & 0\end{array}$ & & & & $4 \% 3$ & 511 & & \\
\hline & 3515 & $=$ & & & & 525 & & & $\bullet 3.9$ & & & & 497 & 515 & & \\
\hline & 45 & $\rightarrow$ & & & & 524 & & +32 & +2.2 & & & & 499 & 517 & & \\
\hline & $25 \quad 5015$ & $\infty$ & & & & $523]^{-}$ & & & -0.6 & & & & 499 & 517 & & \\
\hline
\end{tabular}


IAFELLA III - (SEquilo circuito XIX:)

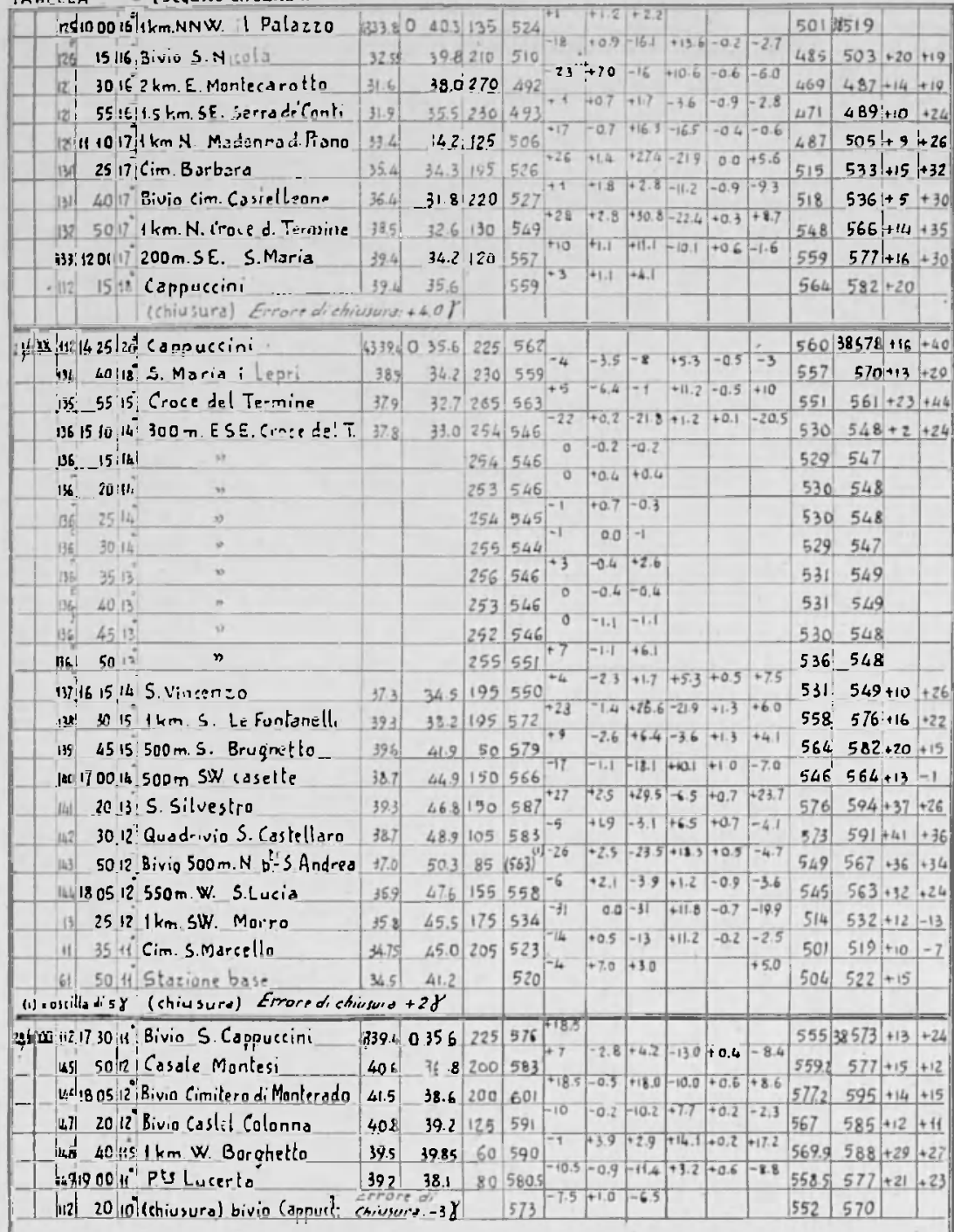

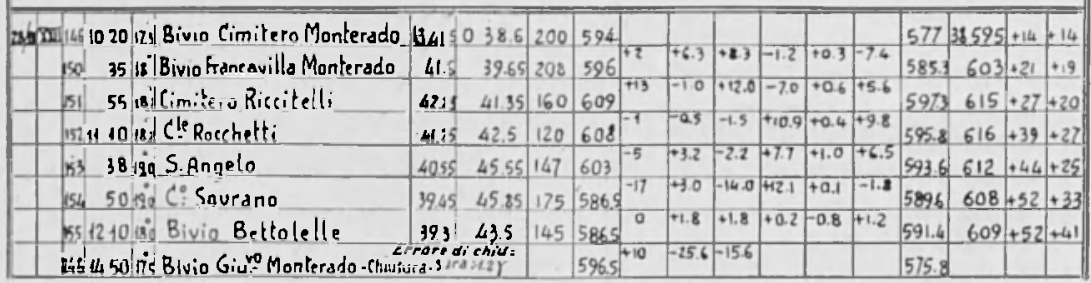


TABELLA "..' (5EGUITO)

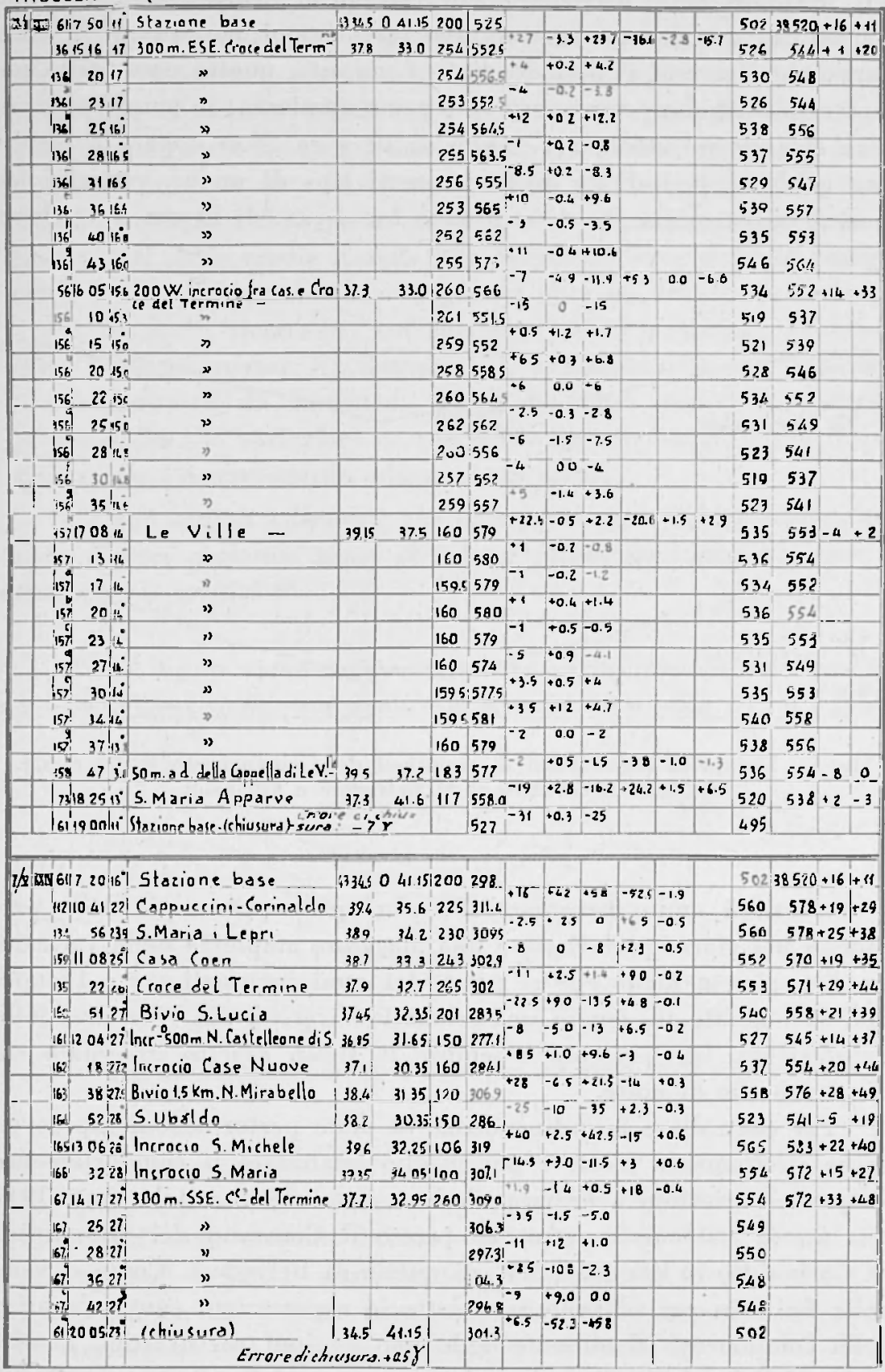


ve tempo (una o due oscillazioni); esse hanno ampiezze molto variabili, maggiori delle precedenti, ed arrivano al massimo a $6 \div 7 \gamma$ ca. Soprattutto spiccano però tra le altre, oscillazioni, che durano a volte oltre un'ora, aventi il periodo di $6 \div 8$ minuti; queste presentano un aspetto più regolare come periodo e come ampiezza; le ampiezze massime si aggirano attorno al valore di $10 \gamma$ ca. Non appare evidente una qualsiasi periodicità di tutti questi tipi di onde; in circa sei mesi di registrazione, se si eccettuano brevi periodi in cui compaiono
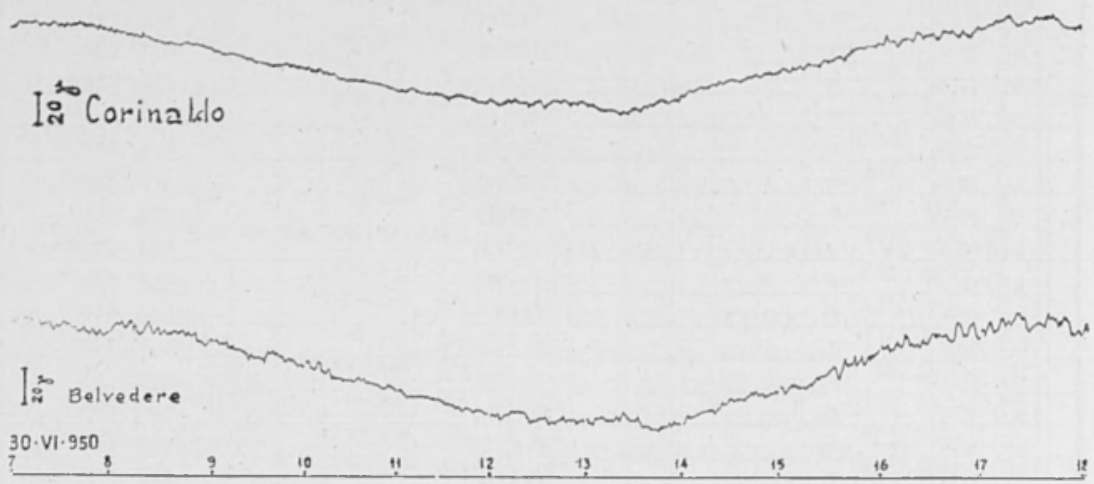

Fig. 5 - Esempi di registrazioni di variazioni della componente $Z$ del c.m.t. ottenute nelle Stazioni di Belvedere e Corinaldo

perturbazioni molto irregolari e notevolmente più ampie delle precedenti nei giorni perturbati, e una maggiore ampiezza della variazione diurna man mano che si passa dai mesi invernali verso l'estate, non si è notata in genere una sensibile diversità sul carattere delle registrazioni, che presentano sempre lo stesso aspetto irregolare sia di gionno che di notte.

Per controllare la indipendenza di dette perturbazioni rapide da cause strettamente locali e per meglio analizzare il comportamento di queste variazioni a breve periodo è stata allestita nel marzo 1949 una nuova stazione registratrice presso il Convento dei Cappuccini di Corinaldo, $12 \mathrm{~km}$ ca. a NW di quella di Belvedere. Come si vede nella fig. 5 in cui vengono riprodotte le registrazioni contemporanee della componente $Z$ ottenute nelle due stazioni registratrici, l'andamento delle variazioni, qualora si tenga conto della diversa sensibilità 
(v. § 2, c) del diverso periodo proprio degli equipaggi nonché del relativo smorzamento, è perfettamente lo stesso in entrambe.

Inoltre l'esame delle registrazioni contemporanee di un declinometro e variometro per la componente orizzontale dell'Osservatorio magnetico di Genova, gentilmente inviateci dal prof. M. Tenani, eseguite con apparecchi aventi una minore sensibilità ha potuto far rilevare la presenza in esse di una analoga microstruttura delle variazioni. Esse sono d'altra parte analoghe a quelle registrate in tutti gli Osservatori delle medie e delle alte latitudini dove si fa la registrazione continua degli elementi magnetici $\left({ }^{7}\right)$ e già furono messe in evidenza da molti ricercatori tra cui per citare i principali Eschehangen $\left({ }^{8}\right)$, Angenbeister $\left({ }^{9}\right)$, Terada $\left({ }^{10}\right)$, Chapman $\left({ }^{11}\right)$, ecc. Esse sembrano provocate da movimenti degli alti strati atmosferici ionizzati in relazione alle variazioni di concentrazione ionica in stretta dipendenza con l'irraggiamento ultravioletto solare.

Si può quindi affermare che comunque sono da escludere per la zona da noi esplorata cause di perturbazioni strettamente locali sia naturali sia artificiali.

Nella fig. 6 viene rappresentata la variazione diurna durante $\mathbf{i}$ giorni della seconda campagna. Anche qui, come nella fig. 4, i grafici,

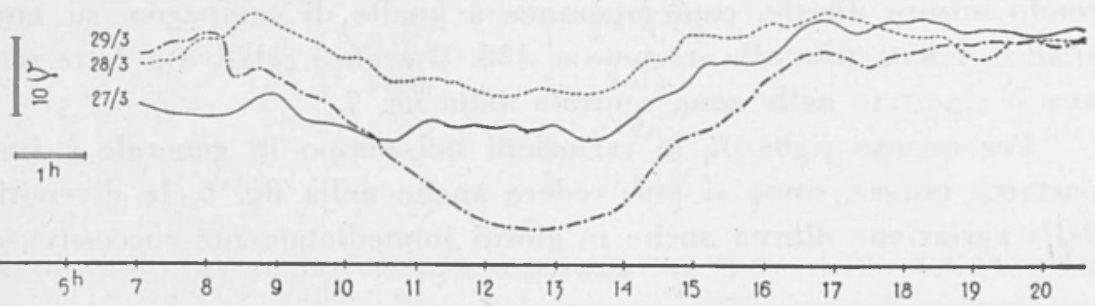

Fig. 6 - Variazione diurna a Belvedere nei giorni 27.29 marzo 1950

sui quali è stata effettivamente computata la correzione delle misure dirette, si sono ottenuti lisciando la curva dei valori diretti delle registrazioni e quindi rappresentano l'andamento medio della variazione diurna. Ciò è stato fatto non solo per la impossibilità pratica di rintracciare sul magnetogramma, la cui scala dei tempi è di 1,65 $\mathrm{cm} / \mathrm{h}$, l'istante corrispondente a quello in cui veniva effettuata la mi- 
sura ma anche perché il valore adottato per ciascuna stazione rappresenta come già si è detto, il valore medio di almeno 8 misure eseguite in tempi diversi (generalmente con intervallo di pochi minuti l'una dall'altra $\left(^{*}\right)$, ma sufficiente per cambiare l'influenza delle variazioni sopramenzionate). Il tempo della misura indicato nelle tabelle è la media di questi tempi arrotondata al minuto.

Abbiamo anche fatto dei tentativi per applicare una correzione per la variazione diurna dedotta direttamente per ogni misura dalla curva non lisciata; ma, data la brevità dello scorrimento e lo spessore del grafico stesso, questa operazione oltre a presentare una difficoltà pratica notevole dà luogo a misure molto incerte.

I risultati ottenuti con questo metodo diretto non danno quindi valori più attendibili di quelli ottenuti con il metodo del lisciamento.

Dall'esame attento delle misure dirette e delle misure corrette per la variazione diurna risulta che gli scarti di pochi $\gamma$ che si riscontrano in alcune stazioni ripetute (v. tab. VII) e qualche sensibile differenza anche nelle misure di stazioni relativamente vicine di uno stesso circuito o di circuiti diversi, debbono attribuirsi alla impossibilità di applicare una perfetta correzione per la variazione diurna piuttosto che ad una eventuale imprecisione delle misure stesse (eseguite sempre con molta cura) oppure ad effettive anomalie locali.

La correzione per la variazione diurna per il giorno 7 maggio è stata ottenuta, al fine di evitare ogni possibile causa di errore, facendo misure dirette, contemporanee a quelle di campagna, su una bilancia tenuta fissa alla stazione $\mathrm{n}$. 135. Il grafico relativo a dette misure è riportato nella zona centrale della fig. 7 .

Per quanto riguarda le variazioni nel tempo in generale $\dot{e} \mathrm{im}$ portante notare, come si può vedere anche nella fig. 6 , le diversità della variazione diurna anche in giorni immediatamente successivi, e

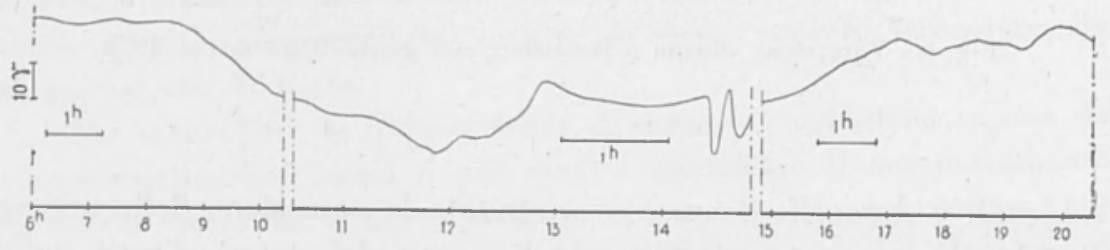

Fig. 7

(*) In media, la distribuzione nel tempo delle 8 misure è così ripartita: le prime quattro misure entro 2 minuti ca. le successive quattro ugualmente entro 2 minuti ca. ma ad un intervallo di $5 \div 6$ minuti dalle prime. 
ciò specialmente nelle ore meridiane, proprio quelle in cui si opera in campagna: per cui la riduzione delle misure non basata su dati contemporanei di una stazione registratrice molto prossima alla zona del rilievo, può portare ad errori anche molto forti (che non è escluso possano ragriungere i $15 \div 20 \gamma$ ).

Si deduce da ciò ancora la necessità di impiantare in Italia un congruo numero di stazioni di registrazione delle variazioni che integrino i dati degli Osservatori Magnetici. Soprattutto per lo studio delle micropulsazioni occorrerebbero variometri con equipaggi di breve periodo opportunamente smorzati, con registratori aventi una scala dei tempi di almeno $12 \mathrm{~cm} / \mathrm{h}$.

5. Precisione del rilievo. - La precisione del rilievo può determinarsi, come è noto, in base agli errori di chiusura dei diversi circuiti.

Tenendo conto del numero di stazioni comprese in ciascun circuito, cioè dando un peso a ciascun errore di chiusura inversamente proporzionale al numero di stazioni relativo a quel circuito, è stato calcolato l'errore medio di ogni singola stazione per le due zone di Iesi e Fermo (f. 117 e 125 della carta topografica 1:100.000) della prima campagna; i calcoli relativi sono riportati nelle tabelle IV e V.

Si sono trovati i seguenti valori:

$$
\begin{array}{r}
m^{\prime}= \pm \sqrt{\frac{\left[\overline{\left.p d^{2}\right]}\right.}{2[p]}}- \pm \sqrt{\frac{\overline{47.2654}}{4.058}}= \pm 3,41 \gamma \\
m= \pm \sqrt{\frac{4.990 y}{0.516}}= \pm 3,92 \gamma
\end{array}
$$

Applicando invece la formula consueta, che tiene conto soltanto del numero $n$ dei circuiti (e non del numero delle stazioni in ogni singolo circuito):

$$
m= \pm \sqrt{\frac{[d \boldsymbol{d}]}{2 n}}
$$

si ottiene per l'errore medio delle stazioni comprese nei fogil 117 e 125 :

$$
m^{\prime}= \pm 3,44 \gamma \quad, \quad m^{\prime \prime}= \pm 3,92 \gamma
$$

L'errore medio di ogni singola stazione è quindi all'incirca doppio di quello che compete ad ogni singola lettura (\$2). 


\section{Tabella IV}

Calcolo dell'errore medio di ogni singola stazione nel $F^{0} 117$ per la prima campagna

\begin{tabular}{|c|c|c|c|c|}
\hline Circuito & $d$ & $d^{2}$ & $p=\frac{1}{n}$ & $p d^{2}$ \\
\hline I & -61 & 3721 & 0125 & 4.6512 \\
\hline II & -6.0 & 36.00 & 0.200 & 7.2000 \\
\hline III & -2.0 & 4.00 & 0.125 & 0.5000 \\
\hline IV & -33 & 1089 & 0.111 & 12100 \\
\hline V & +51 & 26.01 & 0.143 & 3.7157 \\
\hline VI & +3.8 & 14.44 & 0.143 & 20629 \\
\hline VII & -3.9 & 1521 & 0.143 & 21729 \\
\hline VIII & -40 & 1600 & 0.200 & 32000 \\
\hline IX & -6.8 & 4624 & 0.143 & 6.6123 \\
\hline $\mathrm{X}$ & -6.2 & 38.44 & 0,100 & 3.8440 \\
\hline XI & -5.7 & 3249 & 0200 & 6.4 .980 \\
\hline XII & -5.9 & 34.81 & 0.071 & 24864 \\
\hline XIII & +24 & 5.76 & 0125 & 0.7200 \\
\hline \multirow[t]{2}{*}{ XIV } & -36 & 12.96 & 0.200 & 2.5920 \\
\hline & & {$\left[d^{2}\right]=330.46$} & {$[p]=2.029$} & {$\left[p d^{\circ}\right]=472654$} \\
\hline
\end{tabular}

Tabella V

Calcolo dell'errore medio di ogni singola stazione nel Fo 125

\begin{tabular}{|l|r|r|r|r|}
\hline Circuito & $d$ & \multicolumn{1}{|c|}{$d^{2}$} & $p=\frac{1}{n}$ & \multicolumn{1}{c|}{$d F^{2}$} \\
\hline $\mathrm{XV}$ & -1.2 & 144 & 0091 & 0.1309 \\
$\mathrm{XVI}$ & -5.4 & 2916 & 0167 & 4.8600 \\
& & {$[p]=0.258$} & {$\left[p d^{2}\right]=4.9909$} \\
\hline
\end{tabular}


La precisione clie compete alla seconda campagna risulta invece dalla tabella VI che segue:

\section{Tabella VI}

Calcolo dell'errore medio di ogni singola stazione per la seconda campagna (marzo-maggio 1950)

\begin{tabular}{|c|c|c|c|c|}
\hline Circuito & $d$ & $d 2$ & $p=\frac{1}{n}$ & $p d^{2}$ \\
\hline XVII & -5.0 & 25.00 & 0056 & 1.4000 \\
\hline XVIII & -39 & 15.21 & 0.083 & 12624 \\
\hline XIX & +4.0 & 1600 & 0048 & 0.7680 \\
\hline$X X$ & +20 & 4.00 & 0.046 & 0.1840 \\
\hline XXI & -30 & 9.00 & 0.167 & 1.5030 \\
\hline XXII & -1.2 & 1,44 & 0.143 & 02059 \\
\hline XXIII & -7.0 & 49.00 & 0.033 & 1.6170 \\
\hline \multirow[t]{2}{*}{ XXIV } & +05 & 0.25 & 0,063 & 0.0158 \\
\hline & & {$\left[d^{2}\right]=11990$} & {$[p]=+0.639$} & {$[p d \Sigma]=6.9561$} \\
\hline
\end{tabular}

da cui si ricava:

$$
m= \pm 2,34 \gamma
$$

Per controllo, durante la seconda campagna sono state ripetute alcune stazioni già occupate nella prima campagna; le differenze risultano dalla Tab. VII.

Come si vede, queste differenze rientrano nei limiti della precisione degli strumenti con cui si è lavorato, (secondo quanto esposto dalla fine del $\$ 2$, tenendo conto anche dell'ulteriore incertezza derivante dalla variazione diurna (di cui si è discusso al § 4). Le anomalie per queste stazioni sono calcolate sulla base del valore medio.

Un altro controllo della precisione che compete alla seconda campagna si ba dal confronto dei collegamenti fra le stazioni 61 e 112, effettuati più volte con $i$ seguenti risultati: 


\begin{tabular}{|c|c|c|c|c|c|c|c|}
\hline circuito & XVII & $(27$ & marzo & 1950) & : & + & $53,3 \gamma$ \\
\hline ) & XIX & $(28$ & ) & ) ) & $\bullet$ & + & 61,5 . \\
\hline$)$ & $\mathbf{X X}$ & $(28$ & ) & $\Rightarrow$ ) & 8 & + & 56,7 . \\
\hline ) & XXIV & $(7$ & maggio & $\geqslant 1$ & 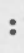 & + & 58,0 , \\
\hline & & ( 8 & 》 & $\begin{array}{c}\text { ) } \\
\text { media }\end{array}$ & & $\begin{array}{l}+ \\
+\end{array}$ & $\begin{array}{l}60,0 \\
57,8\end{array}$ \\
\hline
\end{tabular}

Alla stazione n. 112 è stato quindi attribuito il valore (convenzionale) di $560 \gamma$, e gli errori di chiusura dei circuiti XVII, XIX, XX sono calcolati appunto quale scostamento da questo valore medio.

\section{Tabella VII}

Differenze in $\gamma$ fra $i$ valori della seconda e della prima campagna, per alcune stazioni ripetute

\begin{tabular}{|c|c|c|c|}
\hline Staz. $\mathrm{n}^{0}$ & Differenze & S'az. $\mathrm{n}^{0}$ & Differenze \\
\hline 2 & -1 & 136 & -4 \\
3 & +10 & , a & +1 \\
11 & +9 & , b & -4 \\
13 & +10 & , c & +8 \\
34 & +3 & , d & +8 \\
62 & -2 & , e & -2 \\
73 & -2 & , f & +8 \\
134 & +13 & , g & +5 \\
135 & +2 & , h & +10 \\
& & &
\end{tabular}

6. Valori assoluti. - Per attribuire ai risultati delle misure valori assoluti, ci siamo appoggiati ai valori delle quattro stazioni assolute effettuate nella zona di Iesi (F. 117) dall'Istituto Geografico Militare (contornate con un quadratino nella fig. 1). Nella tabella che segue sono riportati i valori osservati dall'I.G.M. $(I, H)$, ridotti al 1935,0 ed il valore della $Z$ calcolato in corrispondenza, e successivamente ridotto al 1942,0 (epoca per cui è stato calcolato il campo normale della 
$Z$ in Italia) sulla base delle variazioni registrate all'Osservatorio Magnetico di Genova $(Z 1942,0-Z 1935,0=+0,00218$ ).

Nella penultima colonna è riportato pure il valore della $Z$ ricavato da una compensazione fra misure assolute e misure variometricbe, nella quale si è imposta la condizione dell'annullarsi delle reciproche differenze (ultima colonna).

\begin{tabular}{|c|c|c|c|c|c|c|}
\hline \multirow{3}{*}{$\begin{array}{l}\text { Staz. } \\
\text { n. }\end{array}$} & \multicolumn{3}{|c|}{$\begin{array}{c}\text { Valori asroluti } 1 S 35,0 \\
\text { (I.G.M) }\end{array}$} & \multirow{3}{*}{$\begin{array}{c}Z \\
1942,0\end{array}$} & \multirow{3}{*}{$\begin{array}{c}\text { Valori } \\
\text { variome- } \\
\text { trici }\end{array}$} & \multirow{3}{*}{$\begin{array}{c}\text { Differenze } \\
(\gamma)\end{array}$} \\
\hline & \multicolumn{2}{|c|}{ osservati } & calcolato & & & \\
\hline & I & H & Z & & & \\
\hline 17 & $59.27^{\prime}$ & 0.22646 & 038369 & 0,38587 & 0,38540 & +47 \\
\hline 82 & 15 & 725 & 197 & 415 & 436 & -21 \\
\hline 31 & 14 & 694 & 120 & 338 & 406 & -68 \\
\hline 68 & 27 & 633 & 347 & 565 & 522 & +43 \\
\hline
\end{tabular}

Poiché (da quanto precede) è lecito attribuire la maggior parte degli scarti ora individuati alle stazioni assolute, si ha così un'altra prova della insufficiente precisione attuale dei valori della $Z$ ricavati indirettamente dalle misure di $I$ ed $H$ mediante la formula: $Z=H \cdot t g I$, e della necessità di seguire altri criteri nella determinazione di questa componente del campo magnetico terrestre $\left({ }^{4}\right)$.

7. Variazioni normali nello spazio. Anomalie regionali. - Mediante la formula che esprime la distribuzione normale della componente magnetica verticale in Italia al 1942,0 $\left({ }^{6}\right)$ :

$$
\begin{gathered}
Z_{\mathbf{n}}=37810,19+10,9624 .\left(\varphi^{\prime}-2550^{\prime}\right)-0,2472 . \lambda^{\prime}+0,000464 \\
.\left(\varphi^{\prime}-2550^{\prime}\right)^{2}-0,001334\left(\varphi^{\prime}-2550^{\prime}\right) . \lambda^{\prime}-0,000642 \lambda^{\prime 2}
\end{gathered}
$$

dove $\varphi^{\prime}, \lambda^{\prime}$ sono le coordinate geografiche del punto considerato espresse in primi, con la longitudine riferita al meridiano di Roma, abbiamo calcolato anzitutto il valore normale $Z_{n}$ per le stazioni prima e penultima di ogni circuito; e poi dalla prima stazione di ogni circuito si sono ricavati $i$ valori normali nelle altre in funzione delle diffe- 
renze di latitudine e di longitudine, sulla base dei seguenti valori per i gradienti normali $\left({ }^{5}\right)$ :

$$
\begin{array}{lll}
-5,95 & \gamma / \mathbf{k m} \text { di latitudine verso nord, } \\
+0,25 & \gamma / \mathbf{k m} \text { di longitudine verso est. }
\end{array}
$$

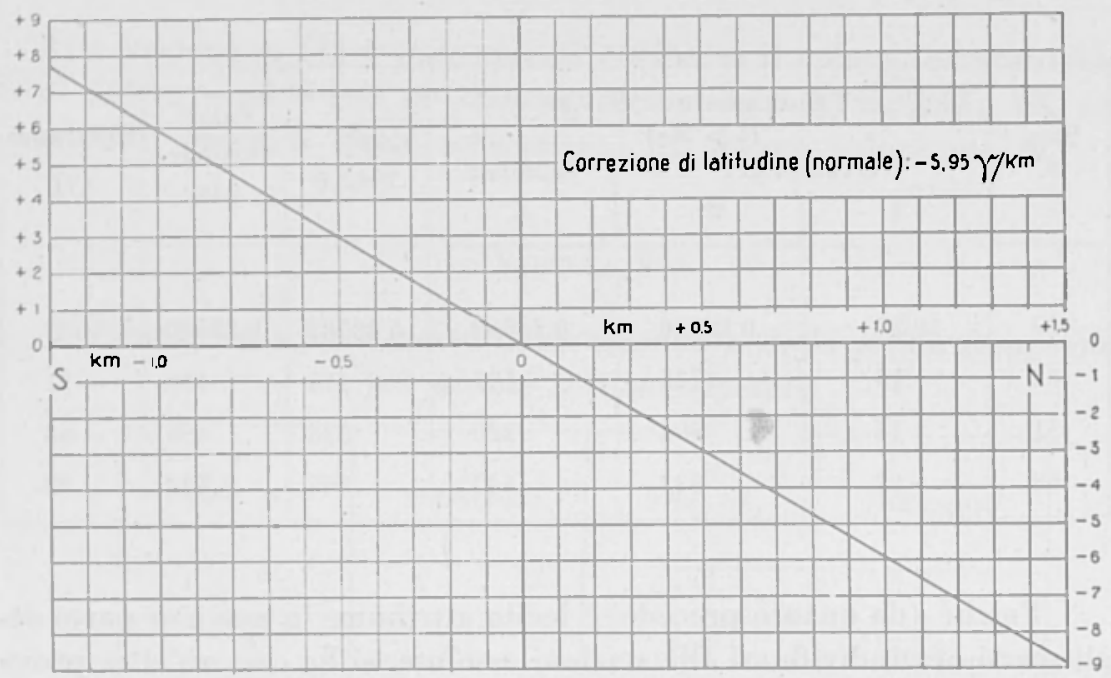

Fig. 8 - Correzione di latitudine per la componente verticale del c. m.t.

Le differenze fra $i$ valori assoluti osservati ed $i$ valori normali così calcolati, hanno fornito $\mathrm{i}$ valori delle anomalie normali $\Delta Z_{\mathrm{n}}$ riportati nelle tabelle I, II e III e graficamente nelle figure 10 e 12.

Come si vede, nella zona di lesi il campo presenta un'anomalia regionale positiva molto spiccata verso $\mathrm{E}$ : essa potrebbe indicare l'avvicinarsi alla superficie di quegli strati sotterranei, a suscettività magnetica più elevata, che danno origine alla cospicua anomalia regionale già messa in evidenza a SE di Ancona dalla rappresentazione vettoriale della componente orizzontale corrispondente alla perturbazione totale del campo magnetico terrestre in Italia $\left({ }^{2}\right)$. .E del resto la presenza in questa zona del Monte Conero, caratteristico massiccio costituito da terreni cretaceo-paleogenici, dimostra chiaramente che l'ampiezza dell'anticlinale è molto cospicua, tanto da far emergere appunto terreni della serie mesozoica.

A scopo puramente illustrativo abbiamo riportato nella fig. 13 
una sezione, da Piagge a Montecassiano, in cui è schematicamente indicata accanto alla topografia superficiale, l'anomalia regionale ora discussa. Nella stessa fig. 13 è riportato anche l'andamento locale dell'anomalia, secondo quanto vedremo nel $\S 8$.

Nella fig. 10 sono poi ancora degne di menzione alcune anomalie locali fra cui tre positive: una nella zona di Belvedere Ostrense, un'altra nella zona a S di Mondavio e la terza in quella di S. Silvestro,

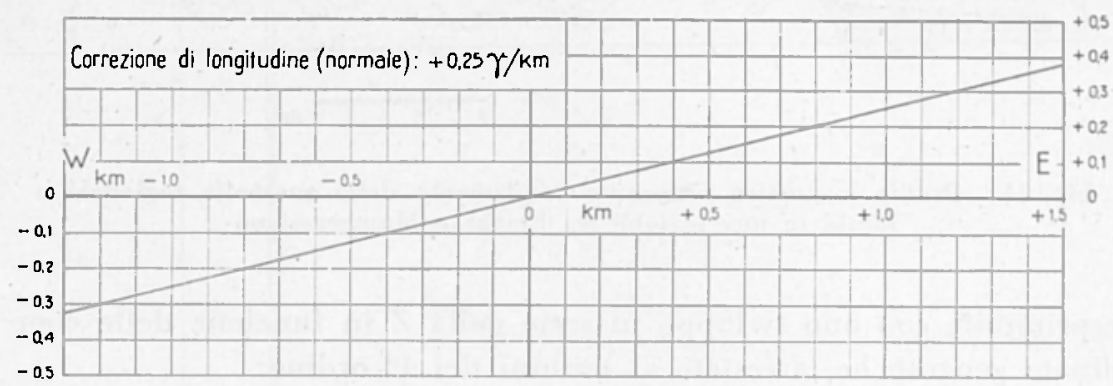

Fig. 9 - Correzione di longitudine per la componente verticale del c.m.t.

e altra negativa nella zona di Corinaldo, Montecarotto e Cupramontana. Le prime si possono imputare probabilmente a qualche dorsale sotterranea, molto limitata in potenza, delle stratificazioni sopra menzionate; mentre le anomalie negative forse fanno parte di una anomalia più estesa, conseguente alla sinclinale in corrispondenza degli strati di terreni eo-miocenici superficiali.

Le isanomale nella zona di Fermo (fig. 12) presentano invece una spiccata anomalia regionale positiva verso sud ed un'anomalia positiva non meglio precisata a $\mathrm{N}$ di Fermo. Dal punto di vista regionale il campo sembra qui un po' più movimentato che nella zona a NW di Iesi, anche se l'esiguo numero di misure eseguite in questa zona non consente di entrare in maggiori particolari.

8. Campo normale locale. Anomalie locali. - Di particolare interesse, specie ai fini della presente ricerca, era però mettere in evidenza le anomalie locali, specie nella regione attorno a Iesi, dove ii rilievo magnetico è stato più dettagliato. A tale fine è stato necessario calcolare anzitutto il campo locale della $Z$ nella regione considerata. Data la limitata estensione di questa, esso si può pensare 


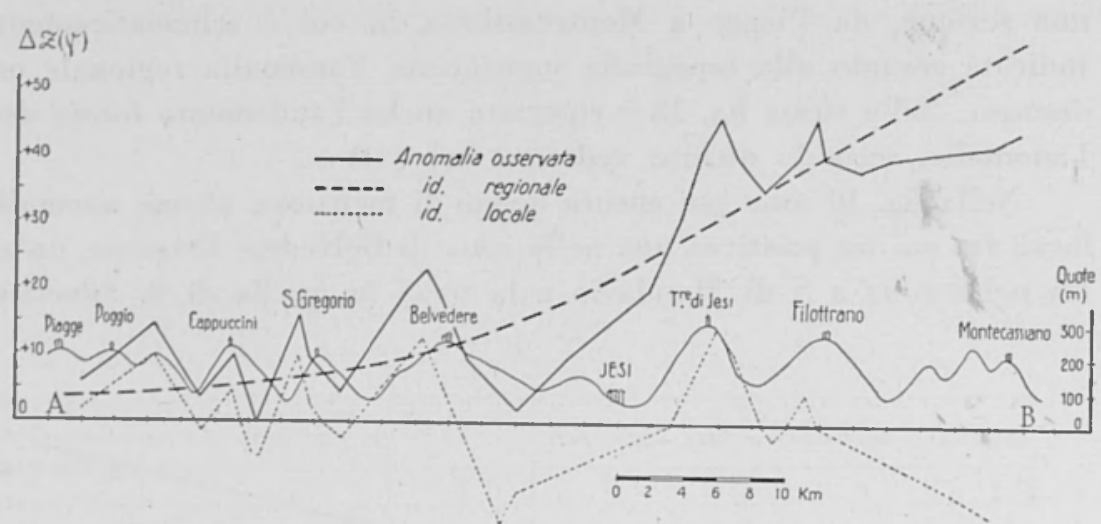

Fig. 11 - Profilo magnetico mostrante l'andamento della anomalia regionale e locale in una sezione tra Piagge e Montecassiano

esprimibile con uno sviluppo in serie della $Z$ in funzione delle coordinate geografiche, arrestate ai termini del $1^{\circ}$ ordine:

$$
Z=Z_{0}+z_{1} \Delta \varphi+z_{2} \Delta \lambda,
$$

dove con $Z_{0}=Z\left(\varphi_{0}, \lambda_{0}\right)$ indichiamo il valore della $Z$ in un punto centrale di riferimento, e con $\Delta p=p-\tau_{0}, \Delta \lambda=\lambda-\lambda_{0}$ le differenze fra le coordinate geografiche dei singoli punti e quelle del punto centrale. Come tale è stato preso il punto

$$
r_{\mathrm{o}}=43^{\circ} 30^{\prime} \mathrm{N}, \lambda_{\mathrm{o}}=0^{\circ} 45^{\prime} \mathrm{E} \text {, }
$$

che corrisponde al centro del f. 117 (Iesi).

Scritta allora la [2] nella forma

$$
Z_{0}+z_{1} \Delta \varphi+z_{2} \Delta \lambda-Z=0
$$

per tutte le 89 stazioni della prima campagna nella regione considerata, e per i valori convenzionali di $Z$ di cui alla fig. I (dato che interessano solo valori relativi) ne è risultato un sistema di 89 equazioni nelle tre incognite $Z_{0}, z_{1}$ e $z_{2}$. Risolto col metodo dei minimi quadrati, esso ba dato luogo al seguente sistema normale:

$$
\begin{array}{r}
89 . Z_{0}+146,25 . z_{1}+132,15 . z^{2}-42096=0 \\
2295,44 . z_{1}-1440,50 . z_{2}-87683,85=0 \\
3516,02 . z_{2}-52720,9=0
\end{array}
$$




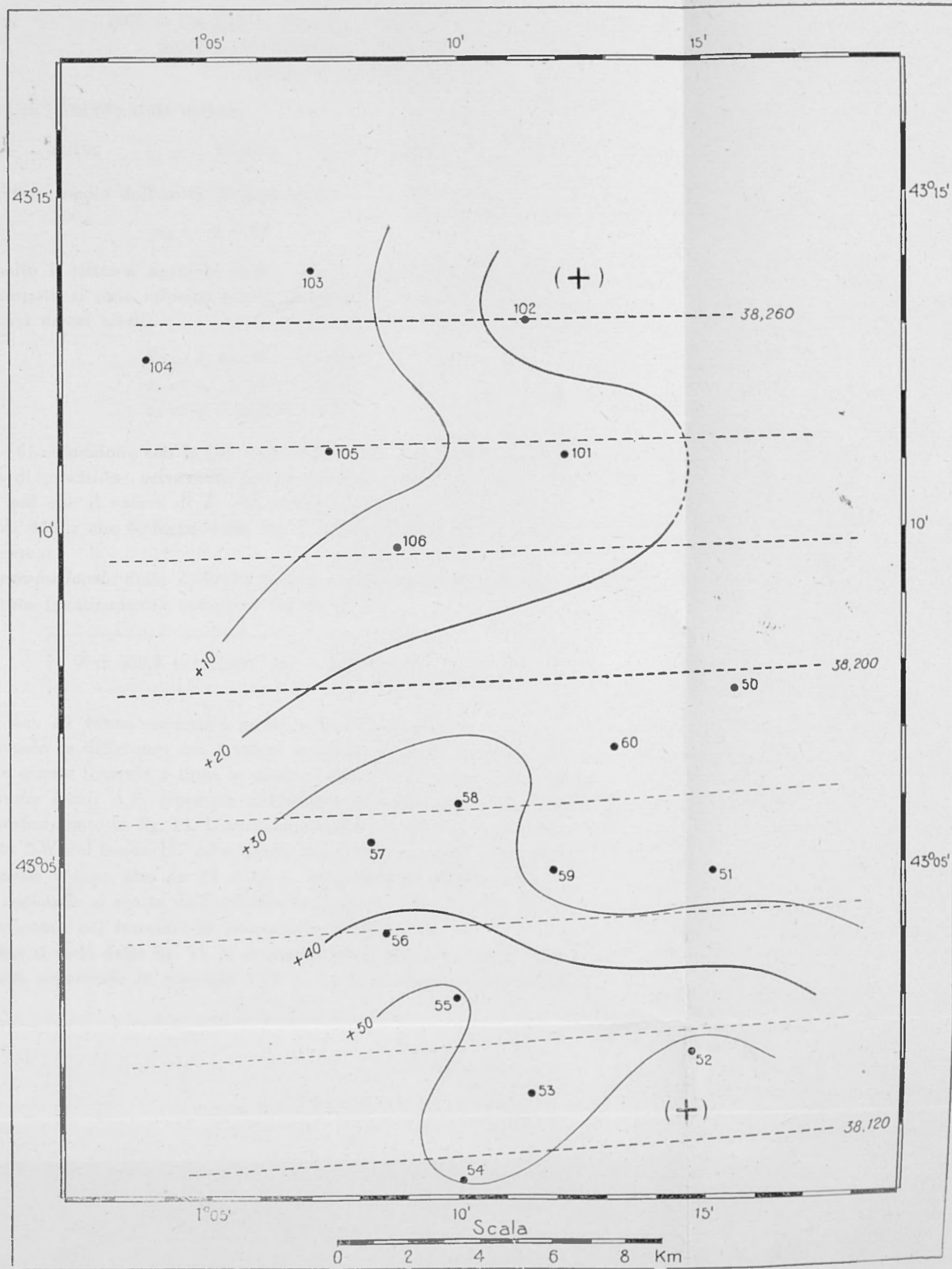

Fig. 12 - Isodinamiche verticali normali (equidistanza $200 \gamma$ ) e isoanomale verticali regionali nella zona di Fermo (equidistanza $6 \gamma$ ). 
da cui si ricava il seguente sistema risolvente:

$$
\begin{array}{r}
89 Z_{0}+146,25 z_{1}+132,15 z_{2}-42096-=0 \\
2055,11 z_{1}-1657,66 z_{2}-18509,24=0 \\
1982,896 z_{2}-5145,040=0
\end{array}
$$

$\operatorname{con}[v v]=7.624,69 ; \mathrm{e}$ da questo:

$$
Z_{0}=+450,90, z_{1}=+11,0991, z_{2}=+2,5947 .
$$

L'errore medio dell'unità di peso risulta

$$
m_{0}=+9,42 \text {. }
$$

Risolto il sistema normale anche con il metodo dei coefficienti indeterminati, si sono ottenuti per le incognite $i$ seguenti valori, con i rispettivi errori medi:

$$
\begin{aligned}
& Z_{0}=+450,90+1,21 \\
& z_{1}=+11,1014+0,27 \\
& z_{2}=+2,5943+0,21
\end{aligned}
$$

Le [6] coincidono con le [4] a meno di 0,003 , e questo è appunto l'ordine di precisione occorrente per le incognite nello sviluppo [2]. Si noti poi che il valore di $Z_{0}$ qui ricavato è in buon accordo con quello di $449 \gamma$ che si legge dalla fig. 1 in corrispondenza al punto considerato.

Il campo locale della $Z$ risulta quindi (valori relativi) sulla zona considerata (praticamente compresa nel f. 117):

$$
Z=450,9+11,1003 \Delta \varphi^{\prime}+2,5945 . \Delta \lambda^{\prime}
$$

dove le $\Delta \varphi, \Delta \lambda$ vanno espresse i primi, e le $Z$ risultano in $\gamma$.

Facendo la differenza fra i valori osservati e quelli ottenuti applicando questa formula a tutte le stazioni del f. 117 , si sono ottenute le anomalie locali $\Delta Z_{1}$ riportate nell'ultima colonna delle Tab. I e III, e graficamente in fig. 13. L'estensione della [7] alle stazioni della estremità NW del foglio 117 ed a quelle del f. 1$] 0$, fornisce valori per le anomalie troppo alte da 10 a $20 \gamma$, in quanto in quella zona il campo regionale si scosta dall'andamento piano (v. fig. ll). Di ciò si è tenuto conto nel tracciare le isoanomale locali in fig. 13 .

Come si vede dalla fig. 13 , le anomalie locali non sono molto sensibili, non superando in generale i $20 \gamma$. La loro origine è probabil- 
mente dovuta a deboli pieghe negli strati sottostanti al Piacenziano ed all'Astiano. Solo in due casi si riscontrano due anomalie locali positive un po' più sensibili ( $30 \gamma$ ), a Sud di Mondavio e nella zona di S. Silvestro; di esse si è però già parlato al $\S 7$.

9. Conclusione. - Da quanto precede risulta chiaramente che la zona investigata non presenta in generale anomalie locali degne di rilievo, che possano sconsigliarla per la scelta di un Osservatorio magnetico con le caratteristiche desiderate. Ai bordi della zona sono bensì manifeste alcune anomalie locali positive piuttosto sensibili ed una cospicua anomalia regionale è manifesta verso $\mathrm{SE}$, ma il loro carattere non si ritiene sia tale da pregiudicare queste conclusioni. Ad ogni modo, come risulta dalla fig. 10 e 13 ed ancor meglio dalla fig. 11 , nella zona da Belvedere a Poggio queste hanno valori (assoluti) minimi, per cui sembra lecito concludere che è in questa zona che converrebbe scegliere la località definitiva per l'Osservatorio.

$$
\text { Roma - Istituto Nazionale di Geofisica - Maggio } 1950 .
$$

\section{RIASSUNTO}

Da vari anni l'Istituto Nazionale di Geofisica ha compiuto campagne magnetiche sistematiche un po' dovunque nell'Italia centrale allo scopo di ricercare una zona geologicamente non perturbata e esente da anomalie magnetiche locali (e possibilmente anche regionali) adatta alla fondazione di un Osservatorio Magnetico di prim'ordine. I risultati di queste ricerche ed indagini, hanno indicato le Marche come regione rispondente allo scopo. In questa nota vengono riportati $i$ risultati del rilievo magnetico regionale ivi eseguito. Essi confermano che la zona investigata non presenta in generale anomalie locali degne di rilievo, che possano sconsigliarla per la scelta di un Osservatorio Magnetico con le caratteristiche desiderate. Ai bordi della zona si sono messe in evidenza alcune anomalie locali positive ed una anomalia regionale verso $\mathrm{SE}, \mathrm{ma}$ esse non pregiudicano le conclusioni favorevoli sopraddette; comunque nella zona tra Belvedere e Poggio le anomalie hanno valori minimi per cui proprio in questo territorio si ritiene opportuno scegliere la località adatta per l'Osservatorio. 


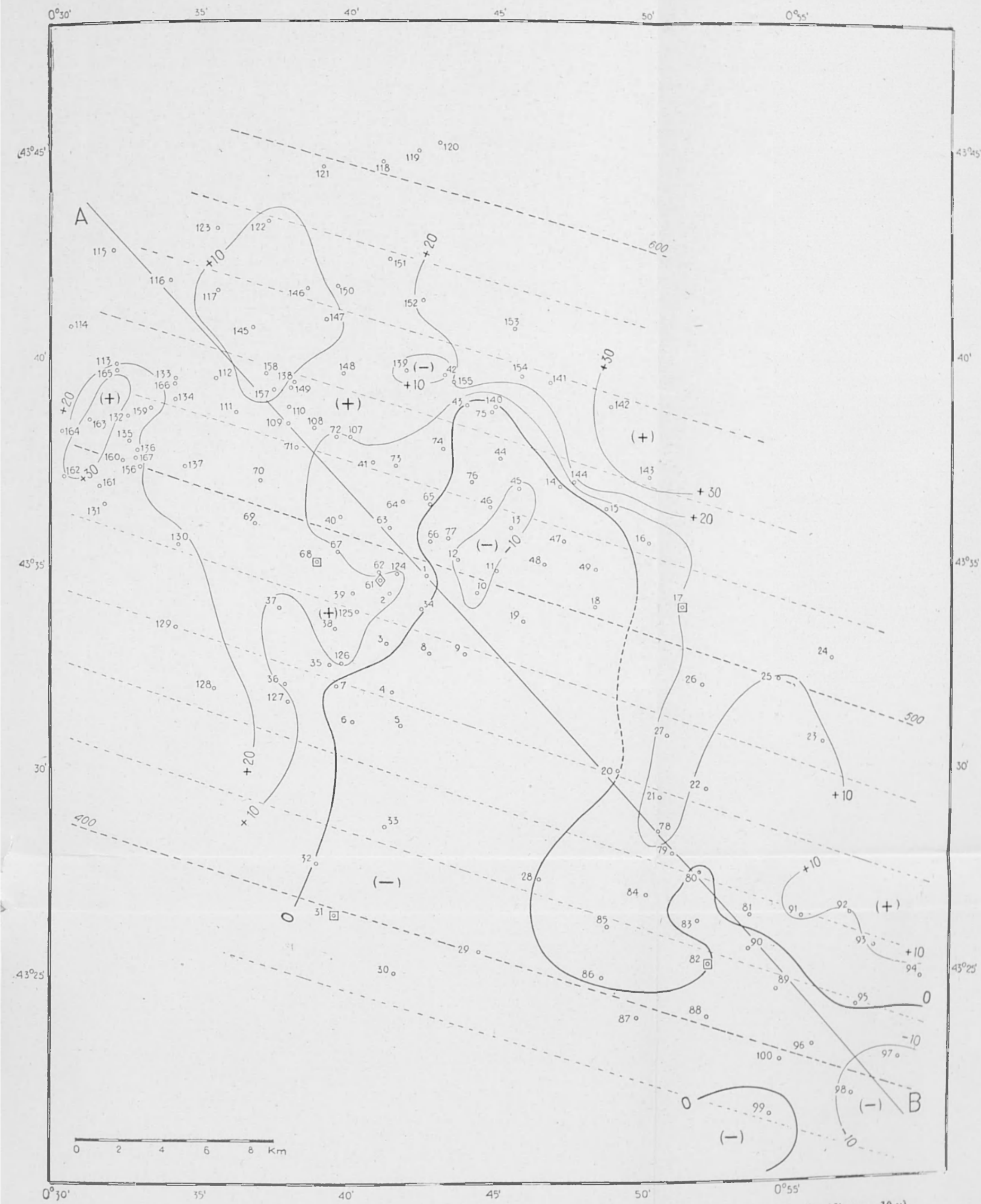

Fig. 13 - Campo normale «locale » nella zona di Jesi (equidistanza $200 \gamma$ ) e anomalie locali della componente verticale (equidistanza $10 \gamma$ ). 


\section{BIBLIOGRAFIA}

(1) Monelli C.: Studio geofisico della regione travertinosa a Sud di Cerdomare (Sabina) - L'Universo, XXV, 1, gennaio 1944.

(2) Motelli C.: La rete geofisica e geodetica in Italia nel suo stato attuale e nei suoi rapporti con la strattura geologica superficiale e profonda - Sez. $1^{\mathrm{a}}$ : Rete magnetica - Tecnica Italiana, I, 1, Trieste 1946; Pubbl. I.N.G. n. 121.

(3) Sacco F. - Bonorelli G.: Note illustrative della Carta Geologica d'Italia. Foglio di Ancona, Jesi, Fermo e Macerata - Roma.

(4) Morelli C.: Nuovi criteri per la sistematica magnetica - Annali di Geofisica, III, 2, Roma 1950.

(5) Morelli C.: Teoria e pratica dei variometri magnetici da campagna - Del Bianco ed., Udine 1947.

(6) Morelli C.: Campo normale della componente verticale del campo magnetico terrestre in Italia - Rivista Geomineraria n. 4, Milano 1943.

(7) Department of Terrestrial Magnetism: The Geomagnetic Field, Description and Analysis - Carnegie Institution of Washington Publ. n. 580. Washington D. C. 1947.

(8) Eschenhagen M.: On minule, periodic changes of the Earth's magnetism - Terr. Magn. 2, 105-114 (1897).

(9) Angeneister S.: Ueber die Fortpflanzungs-geschwindigkeit erdmagnetischer Stoerungen und Pulsationen - Terr. Magn. 25, 26-32 (1920).

(10) Terada T.: On rapid periodic variations of terrestrial magnetism - J. Coll. Sci. Imp. Univ, Tokyo, 37, 1-85.

(11) Chapman S. and Bartels J.: Geomagnetism - Oxford 1940. 